\title{
Asymptotic Representations of Quantum Affine Superalgebras
}

\author{
Huafeng ZHANG
}

Departement Mathematik and Institut für Theoretische Physik, ETH Zürich, Switzerland E-mail: huafeng.zhang@math.ethz.ch

Received April 21, 2017, in final form August 17, 2017; Published online August 22, 2017 https://doi.org/10.3842/SIGMA.2017.066

\begin{abstract}
We study representations of the quantum affine superalgebra associated with a general linear Lie superalgebra. In the spirit of Hernandez-Jimbo, we construct inductive systems of Kirillov-Reshetikhin modules based on a cyclicity result that we established previously on tensor products of these modules, and realize their inductive limits as modules over its Borel subalgebra, the so-called $q$-Yangian. A new generic asymptotic limit of the same inductive systems is proposed, resulting in modules over the full quantum affine superalgebra. We derive generalized Baxter's relations in the sense of Frenkel-Hernandez for representations of the full quantum group.
\end{abstract}

Key words: quantum groups; superalgebras; asymptotic representations; Baxter operators

2010 Mathematics Subject Classification: 17B37; 17B10; 81R50

\section{Introduction}

Let $q$ be a non-zero complex number which is not a root of unity. Let $\mathfrak{g}:=\mathfrak{g l}(M, N)$ be the general linear Lie superalgebra. Let $U_{q}(\widehat{\mathfrak{g}})$ be the associated quantum affine superalgebra (Definition 3.1). This is a Hopf superalgebra neither commutative nor co-commutative, and it can be seen as a deformation of the universal enveloping algebra of the affine Lie superalgebra $\widehat{\mathfrak{g}}:=\mathfrak{g} \otimes \mathbb{C}\left[t, t^{-1}\right]$ of central charge zero without derivation. In this paper we study a certain one-parameter family of infinite-dimensional representations of $U_{q}(\widehat{\mathfrak{g}})$, which arise from suitable limits of finite-dimensional irreducible modules, the so-called Kirillov-Reshetikhin modules.

1. Background. Our study of quantum affine superalgebras is inspired, on the one hand, from the integrability structure of supersymmetric models (AdS/CFT, Hubbard model, etc.; see for example [8]), and on the other hand, from the representation theoretical interpretation of transfer matrices for quantum integrable systems.

In the early seventies, towards the study of the transfer matrix $\mathbf{T}$ of the eight-vertex model, Baxter [1] introduced $\mathbf{Q}$-operators and $\mathbf{T}-\mathbf{Q}$ functional relations to solve the spectra of $\mathbf{T}$. Within the framework of quantum inverse scattering method, the representation meaning of Q-operators and T-Q relations was clarified in a series of papers [2, 4, 5, 6] for lattice models and quantum field theories whose symmetry algebras are the quantum affine algebras attached to $\mathfrak{s l}_{2}, \mathfrak{s l}_{3}$. The idea goes roughly as follows. The affine quantum group $U_{q}\left(\widehat{\mathfrak{s l}_{2}}\right)$ admits a universal $R$-matrix $\mathcal{R} \in \mathfrak{B}_{+} \otimes \mathfrak{B}_{-}$with $\mathfrak{B}_{ \pm}$Borel subalgebras. One fixes the so-called quantum space, a representation $W$ of $\mathfrak{B}_{-}$provided by the integrable models. This defines an L-operator, an element of a completed tensor product $\mathfrak{B}_{+} \otimes$ End $W$. The $\mathbf{T}, \mathbf{Q}$-operators, as elements of End $W$, are twisted traces of $\mathbf{L}$ over various representations of $\mathfrak{B}_{+}$: finite-dimensional evaluation representations over $U_{q}\left(\widehat{\mathfrak{s l}_{2}}\right)$ for $\mathbf{T}$ and oscillator representations for $\mathbf{Q}$. Baxter's $\mathbf{T}-\mathbf{Q}$ relations are then deduced from tensor product decompositions of representations of $\mathfrak{B}_{+}$.

The oscillator representations of Borel subalgebras were subsequently extended to Lie superalgebras: $\mathfrak{s l}(2 \mid 1)$ in [7]; $\mathfrak{g l}(M, N)$ in [31]; twisted case of $\mathfrak{o s p}(1 \mid 2)$ in [22, 24]. In these works, one of the main ingredients is the oscillator realization of Borel subalgebras. 
The recent work [3] on hypergeometric equations asks for similar T-Q formulations for the exceptional Lie superalgebra $D(2,1 ; \alpha)$. The associated quantum affine superalgebra is still less understood in aspects of structure/representation theory, beyond the Drinfeld loop realizations [18]. Its universal $R$-matrix remains unknown.

In [21], Hernandez-Jimbo proposed the oscillator representations of Borel subalgebras for an arbitrary non-twisted quantum affine algebra $U_{q}(\widehat{\mathfrak{a}})$. Their main idea is to take a suitable inductive limit of a distinguished family of finite-dimensional $U_{q}(\widehat{\mathfrak{a}})$-modules, the Kirillov-Reshetikhin modules. The limit construction enabled Frenkel-Hernandez [14] to derive generalized T-Q relations in terms of representations and to solve a conjecture of Frenkel-Reshetikhin on the spectra of quantum integrable systems [16].

In this paper, we extend Hernandez-Jimbo's limit construction to the quantum affine superalgebra $U_{q}(\widehat{\mathfrak{g}})$. The Borel subalgebra in our situation will be the $q$-Yangian $Y_{q}(\mathfrak{g})$. Furthermore we perform a new limit, generic asymptotic limit, to the inductive system of Kirillov-Reshetikhin modules, resulting in modules over $U_{q}(\widehat{\mathfrak{g}})$ itself.

2. A toy example. The generic asymptotic limit in the present paper is best viewed in the case of the finite-type Drinfeld-Jimbo quantum group $U_{q}\left(\mathfrak{s l}_{2}\right)$. This is an associative algebra generated by three elements $e^{+}, e^{-}, K$ subject to relations

$$
K \text { invertible, } \quad K e^{ \pm}=q^{ \pm 2} e^{ \pm} K, \quad e^{+} e^{-}-e^{-} e^{+}=\frac{K-K^{-1}}{q-q^{-1}} .
$$

To each positive integer $k$ is attached a representation of $U_{q}\left(\mathfrak{s l}_{2}\right)$ on $V_{k}:=\oplus_{i=0}^{k} \mathbb{C} v_{i}$ with

$$
K v_{i}=q^{k-2 i} v_{i}, \quad e^{+} v_{i}=\frac{q^{i}-q^{-i}}{q-q^{-1}} v_{i-1}, \quad e^{-} v_{i}=\frac{q^{k-i}-q^{-k+i}}{q-q^{-1}} v_{i+1} .
$$

The matrix entries of $K, e^{ \pm} \in \operatorname{End}\left(V_{k}\right)$ at $\left(v_{i}, v_{j}\right)$ are Laurent polynomials in $q^{k}$ for $k>i+$ $j+1$. Specializing such polynomials to a fixed non-zero complex number $c \in \mathbb{C}^{\times}$, we obtain a representation of $U_{q}\left(\mathfrak{s l}_{2}\right)$ on $V_{\infty}:=\oplus_{i=0}^{\infty} \mathbb{C} v_{i}$ with ${ }^{1}$

$$
K v_{i}=c q^{-2 i} v_{i}, \quad e^{+} v_{i}=\frac{q^{i}-q^{-i}}{q-q^{-1}} v_{i-1}, \quad e^{-} v_{i}=\frac{c q^{-i}-c^{-1} q^{i}}{q-q^{-1}} v_{i+1} .
$$

Roughly speaking $V_{\infty}$ is an analytic continuation of the $\left(V_{k}\right)_{k \in \mathbb{Z}_{>0}}$ with respect to the discrete parameter $q^{k}$ - one replaces $q^{k}$ everywhere by $c$.

3. Main results. Let $I_{0}:=\{1,2, \ldots, M+N-1\}$ be the set of Dynkin vertices of $\mathfrak{g}$. There are $U_{q}(\widehat{\mathfrak{g}})$-valued power series $\phi_{i}^{ \pm}(z)$ in $z^{ \pm 1}$ for $i \in I_{0}$ whose coefficients mutually commute; they can be viewed as $q$-analogs of $A \otimes t^{ \pm n} \in \widehat{\mathfrak{g}}$ with $A$ being a diagonal matrix in $\mathfrak{g}$ and $n$ a positive integer. The $q$-Yangian $Y_{q}(\mathfrak{g})$ contains the coefficients of the $\phi_{i}^{+}(z)$, but not $\phi_{i}^{-}(z)$. There is a highest weight representation theory adapted to a triangular decomposition of $U_{q}(\widehat{\mathfrak{g}})$ whose Cartan part is generated by the $\phi_{i}^{ \pm}(z)$.

Fix $r$ a Dynkin vertex and $a \in \mathbb{C}^{\times}$a spectral parameter. To a positive integer $k$ is attached the Kirillov-Reshetikhin (KR) module, the unique finite-dimensional irreducible $U_{q}(\widehat{\mathfrak{g}})$-module $W_{k}$ which is generated by a highest weight vector $\omega_{k}$ such that ${ }^{2}$

$$
\phi_{r}^{ \pm}(z) \omega_{k}=q_{r}^{k} \frac{1-z a}{1-z a q_{r}^{2 k}} \omega_{k}, \quad \phi_{i}^{ \pm}(z) \omega_{k}=\omega_{k} \quad \text { for } \quad i \neq r
$$

as power series in $z^{ \pm 1}$. Here $q_{r}=q$ for $r \leq M$ and $q_{r}=q^{-1}$ for $r>M$.

\footnotetext{
${ }^{1}$ Let $V$ be a vector space with basis $B$ and let $b, b^{\prime} \in B$. The matrix entry of a linear endomorphism $f \in \operatorname{End}(V)$ at $\left(b^{\prime}, b\right)$ is by definition the coefficient of $b^{\prime}$ in $f(b)$.

${ }^{2}$ In [21] actually $\frac{q_{r}^{k}-z a q_{r}^{-k}}{1-z a}$ was used in the inductive system of KR modules. This is due to opposite triangular properties of the coproduct of the $\phi_{i}^{ \pm}(z)$ between [10, Section 7] and Proposition 6.1.
} 
The $W_{k}$ are affine analogs of the $U_{q}\left(\mathfrak{s l}_{2}\right)$-modules $V_{k}$ above. The uniform choice of bases for them relies on an inductive system of vector superspaces (not of $U_{q}(\widehat{\mathfrak{g}})$-modules)

$$
W_{1} \subseteq W_{2} \subseteq W_{3} \subseteq \cdots, \quad \omega_{1}=\omega_{2}=\omega_{3}=\cdots .
$$

We follow the idea of Hernandez-Jimbo [21], which is a fusion procedure relating different tensor products of KR modules. In the super case we need the main results in our previous paper [34] to validate the fusion (Lemma 4.2).

Let $W_{\infty}=\cup_{k>0} W_{k}$ be the inductive limit. Choose a basis $B_{k}$ of $W_{k}$ inductively on $k>0$ so that $\omega_{1} \in B_{1} \subseteq B_{2} \subseteq B_{3} \subseteq \cdots$. Then $B_{\infty}:=\cup_{k>0} B_{k}$ forms a basis of $W_{\infty}$.

3.1. Asymptotic property. To each triple $\left(x, b, b^{\prime}\right) \in U_{q}(\widehat{\mathfrak{g}}) \times B_{\infty} \times B_{\infty}$ is associated a unique Laurent polynomial $P_{b^{\prime} b}^{x}(u) \in \mathbb{C}\left[u, u^{-1}\right]$ satisfying (Lemma 5.1):

- the matrix entry of $x \in \operatorname{End}\left(W_{k}\right)$ at $\left(b^{\prime}, b\right)$ is $P_{b^{\prime} b}^{x}\left(q_{r}^{k}\right)$ for $k$ large enough; ${ }^{3}$

- given $b$ and $x$, we have $P_{b^{\prime} b}^{x}(u)=0$ for all but finitely many $b^{\prime}$.

Our proof of the asymptotic property is more constructive than [21]. It is based on representation theory of finite-type quantum groups of $\mathfrak{s l}_{2}$ and $\mathfrak{s l}(1,1)$.

3.2. Generic asymptotic limit. Let $c$ be a non-zero complex number, called spin parameter. The representation $\left(\rho_{c}, W_{\infty}\right)$ of $U_{q}(\widehat{\mathfrak{g}})$ is defined by requiring (Corollary 5.2):

- the matrix entry of $x \in \operatorname{End}\left(W_{\infty}\right)$ at $\left(b^{\prime}, b\right)$ is the evaluation $P_{b^{\prime} b}^{x}(c)$.

For example, let $b=\omega_{1}$ and $x=\phi_{r}^{ \pm}(z)$. We have $P_{b^{\prime} b}^{x}\left(q_{r}^{k}\right)=q_{r}^{k} \frac{1-z a}{1-z a q_{r}^{2 k}} \delta_{b^{\prime} b}$ by definition of KR modules. As a power series in $z^{ \pm 1}$ its coefficients are Laurent polynomials in $q_{r}^{k}$. Therefore $\phi_{r}^{ \pm}(z) \omega_{1}=c \frac{1-z a}{1-z a c^{2}} \omega_{1}$ in $\left(\rho_{c}, W_{\infty}\right)$. Similarly $\phi_{i}^{ \pm}(z) \omega_{1}=\omega_{1}$ if $i \neq r$.

The generic asymptotic limit applied to non-twisted quantum affine algebras in the Appendix, we obtain $U_{q}(\widehat{\mathfrak{a}})$-modules $\left(\rho_{c}\right)_{c \in \mathbb{C}^{\times}}$. They belong to the category $\mathcal{O}$ introduced by Hernandez [19] and further studied by Mukhin-Young [25]. If $c \notin \pm q^{\mathbb{Z}}$, then $\rho_{c}$ is irreducible and is a minimal affinization of a parabolic Verma module [25]. Analytic continuation was used to prove genericity properties of minimal affinizations [25].

3.3. Hernandez-Jimbo's limit. The arguments of [21] can be adapted to our situation to get a $Y_{q}(\mathfrak{g})$-module structure on $W_{\infty}$, an oscillator module in [7,31]. One modifies the $Y_{q}(\mathfrak{g})$-module structure on $W_{k}$, by tensoring with a one-dimensional $Y_{q}(\mathfrak{g})$-module, so that $\phi_{i}^{+}(0) \omega_{k}=\omega_{k}$ for all $i \in I_{0}$. In this way one loses the $U_{q}(\widehat{\mathfrak{g}})$-module structure.

The advantage is that $P_{b^{\prime} b}^{x}(u) \in \mathbb{C}[u]$ for each triple $\left(x, b, b^{\prime}\right) \in Y_{q}(\mathfrak{g}) \times B_{\infty} \times B_{\infty}$. We define the representation $\left(\rho_{+}, W_{\infty}\right)$ of $Y_{q}(\mathfrak{g})$ by requiring (Corollary 5.2):

- the matrix entry of $x \in \operatorname{End}\left(W_{\infty}\right)$ at $\left(b^{\prime}, b\right)$ is the evaluation $P_{b^{\prime} b}^{x}(0)$.

Again take $x=\phi_{r}^{+}(z)$ and $b=\omega_{1}$. Then $P_{b^{\prime} b}^{x}\left(q_{r}^{k}\right)=\frac{1-z a}{1-z a q_{r}^{2 k}} \delta_{b^{\prime} b}$, which as a power series in $z$ has as coefficients polynomials in $q_{r}^{k}$. It follows that $\phi_{r}^{+}(z) \omega_{1}=(1-z a) \omega_{1}$ in $\left(\rho_{+}, W_{\infty}\right)$. Similarly $\phi_{i}^{ \pm}(z) \omega_{1}=\omega_{1}$ if $i \neq r$.

Informally, one can think of $\left(\rho_{+}, W_{\infty}\right)$ as $\left(\rho_{0}, W_{\infty}\right)$. Contrary to the non-graded case, $W_{\infty}$ is finite-dimensional $\left(2^{M N}\right)$ for the odd Dynkin vertex $r=M$.

3.4. Generalized Baxter's relations. As in [21] we introduce a monoidal category $\mathcal{O}$ of representations of $Y_{q}(\mathfrak{g})$ including all the finite-dimensional $U_{q}(\widehat{\mathfrak{g}})$-modules, the $\rho_{+}$and $\rho_{c}$. In a fraction ring of the Grothendieck ring of $\mathcal{O}$, the isomorphism class of a finite-dimensional $U_{q}(\widehat{\mathfrak{g}})$-module $[V]$ is a polynomial in the ratios $\frac{\left[\left(\rho_{b}, W_{\infty}\right)\right]}{\left[\left(\rho_{c}, W_{\infty}\right)\right]}$ whose coefficients are isomorphism

\footnotetext{
${ }^{3}$ The $U_{q}(\widehat{\mathfrak{g}})$-module structure on $W_{k}$ defines $x \in \operatorname{End}\left(W_{k}\right)$. When $k$ is large enough $b, b^{\prime} \in B_{k}$ are basis vectors of $W_{k}$, so the matrix entry makes sense.
} 
classes of one-dimensional $U_{q}(\widehat{\mathfrak{g}})$-modules; see Theorem 6.11. This form generalized Baxter's relations à la Frenkel-Hernandez [14].

The key point in the proof is that normalized $q$-characters of the $\left(\rho_{c}, W_{\infty}\right)$ are identical, which is a consequence of the generic asymptotic limit; see Lemma 6.7. Note that the statement of Theorem 6.11 only involves $U_{q}(\widehat{\mathfrak{g}})$-modules.

4. Perspectives. The generic asymptotic limit works for Felder's elliptic quantum groups [12] of which Borel subalgebras are still unknown; see [13, 37]. It should eventually be done for other quantum affine superalgebras like $U_{q}(\widehat{D}(2,1 ; \alpha))$.

In the sequels $[13,36,37]$ we define Baxter $\mathbf{Q}$-operators from transfer matrices of the $\rho_{c}$, and interpret Theorem 6.11 as generalized T-Q relations of transfer matrices. Surprisingly the spin parameter $c$ becomes the spectral parameter of $\mathbf{Q}$-operators.

This paper is organized as follows. Section 2 explains the idea of generic asymptotic construction. In Section 3, we recall basic properties of the quantum affine superalgebra. Section 4 constructs inductive systems of Kirillov-Reshetikhin modules. In Section 5 we carry out in detail the two limit constructions. In Section 6 we introduce category $\mathcal{O}$ and state generalized Baxter's relations, whose proof is completed in Section 7 together with examples. In the appendix we apply our generic asymptotic construction to non-graded quantum affine algebras.

\section{Idea of asymptotic construction}

Throughout this paper, all the vector spaces and algebras are defined over the base field $\mathbb{C}$. For two vector spaces $V, W$, let $\operatorname{Hom}(V, W)$ denote the set of all linear maps $V \longrightarrow W$.

Fix $A$ to be a unital associative algebra. Let $S$ be a system of algebraic generators, so that $A$ is the quotient of the free associative algebra $\mathbb{C}\langle S\rangle$ by the defining ideal $\mathcal{I}_{S}$.

For all positive integer $k \in \mathbb{Z}_{>0}$ let a representation $\rho^{k}: A \longrightarrow \operatorname{End}\left(V_{k}\right)$ of $A$ be given. Let $\left(F_{k, l}: V_{l} \longrightarrow V_{k}\right)_{l<k}$ be an inductive system of vector spaces; namely $F_{k, l} F_{l, m}=F_{k, m}$ for $m<l<k$ as linear maps $V_{m} \longrightarrow V_{k}$. Assume that the $F_{k, l}$ are injective.

Fix $L, K \in \mathbb{Z}_{>0}$. We assume the asymptotic property: for all $l \in \mathbb{Z}_{>0}$ and $s \in S$, there exists a $\operatorname{Hom}\left(V_{l}, V_{l+L}\right)$-valued Laurent polynomial $P_{s ; l}(u)=\sum_{i=-K}^{K} P_{s ; l}^{[i]} u^{i}$ in $u$ such that

$$
\rho^{k}(s) F_{k, l}=\left.F_{k, l+L} P_{s ; l}(u)\right|_{u=q^{k}} \in \operatorname{Hom}\left(V_{l}, V_{k}\right) \quad \text { for } \quad k>l+L .
$$

Let us prove that the Laurent polynomial $P_{s ; l}(u)$ is unique. Indeed, let $Q(u)$ be another such Laurent polynomial and let $D(u)=P_{s ; l}(u)-Q(u)$. Then from the injectivity of $F_{k, l+L}$ we have $\left.D(u)\right|_{u=q^{k}}=0$ for all $k>l+L$. Since $q$ is not a root of unity, a Vandermonde matrix argument shows that $D(u)=0$.

Similarly one shows that for fixed $s \in S$ and $-K \leq i \leq K$, the linear maps $\left(P_{s ; l}^{[i]}\right)_{l>0}$ form a morphism of inductive systems: $F_{l+L, m+L} P_{s ; m}^{[i]}=P_{s ; l}^{[i]} F_{l, m} \in \operatorname{Hom}\left(V_{m}, V_{l+L}\right)$ for $m<l$. Let $P_{s}^{[i]} \in \operatorname{End}\left(V_{\infty}\right)$ be its inductive limit with $V_{\infty}$ being the inductive limit of $\left(V_{l}, F_{k, l}\right)$.

Claim 2.1. Let $c \in \mathbb{C}^{\times}$. Then $s \mapsto \sum_{i=-K}^{K} P_{s}^{[i]} c^{i}$ defines a representation of $A$ on $V_{\infty}$.

The proof is again a Vandermonde matrix argument, and is omitted. As an example, suppose $s, t \in S$ and $s t \in \mathcal{I}_{S}$. Let us show

$$
\left(\sum_{i=-K}^{K} P_{s}^{[i]} c^{i}\right)\left(\sum_{i=-K}^{K} P_{t}^{[i]} c^{i}\right)=0 .
$$


We have

$$
0=\rho^{k}(s t) F_{k, l}=\rho^{k}(s) \underline{\rho^{k}(t) F_{k, l}}=\underline{\rho^{k}(s) F_{k, l+L}} P_{t ; l}\left(q^{k}\right)=F_{k, l+2 L} P_{s ; l+L}\left(q^{k}\right) P_{t ; l}\left(q^{k}\right)
$$

for all $k>l+2 L$. This forces $\left.P_{s ; l+L}(u) P_{t ; l}(u)\right|_{u=q^{k}}=0$ for all $k>l+2 L$ and so $P_{s ; l+L}(u) P_{t ; l}(u)$ $=0$. Taking inductive limit $l \rightarrow \infty$ and $u=c$ leads to the desired identity.

If furthermore the $P_{s ; l}(u)$ are polynomials in $u$, then in Claim 2.1 one can take $c=0$.

\section{Backgrounds on quantum superalgebras}

This section collects basic facts on the RTT realization of the quantum affine superalgebra $U_{q}(\widehat{\mathfrak{g}})$, the $q$-Yangian $Y_{q}(\mathfrak{g})$ and the quantum superalgebra $U_{q}(\mathfrak{g})$ following [34].

Fix $M, N \in \mathbb{Z}_{>0}$. Set $I:=\{1,2, \ldots, M+N\}, \mathbb{Z}_{2}:=\mathbb{Z} / 2 \mathbb{Z}=\{\overline{0}, \overline{1}\}$. For $i \in I$,

$$
|i|=:\left\{\begin{array}{ll}
\overline{0}, & i \leq M, \\
\overline{1}, & i>M,
\end{array} \quad d_{i}:=\left\{\begin{array}{rl}
1, & i \leq M, \\
-1, & i>M,
\end{array} \quad q_{i}:=q^{d_{i}} .\right.\right.
$$

Define the weight lattice $\mathbf{P}:=\oplus_{i \in I} \mathbb{Z} \epsilon_{i}$ with bilinear form $():, \mathbf{P} \times \mathbf{P} \longrightarrow \mathbb{Z},\left(\epsilon_{i}, \epsilon_{j}\right)=\delta_{i j} d_{i}$. Let $|\cdot|: \mathbf{P} \longrightarrow \mathbb{Z}_{2}$ be the morphism of abelian groups such that $\left|\epsilon_{i}\right|=|i|$. Set $I_{0}:=I \backslash\{M+N\}$. For $i \in I_{0}$, let $\alpha_{i}:=\epsilon_{i}-\epsilon_{i+1}$. Define the root lattice $\mathbf{Q}=\oplus_{i \in I_{0}} \mathbb{Z} \alpha_{i} \subset \mathbf{P}$, and root cones $\mathbf{Q}_{+}:=\oplus_{i \in I_{0}} \mathbb{Z}_{\geq 0} \alpha_{i}$ and $\mathbf{Q}_{-}:=-\mathbf{Q}_{+}$.

Only three cases of $|x| \in \mathbb{Z}_{2}$ will be admitted: $x \in I ; x \in \mathbf{P} ; x$ is a $\mathbb{Z}_{2}$-homogeneous vector of a vector superspace $V$. Naturally $\operatorname{Hom}(V, V)=: \operatorname{End}(V)$ is a superalgebra.

Let $\mathbf{V}:=\bigoplus_{i \in I} \mathbb{C} v_{i}$ be the vector superspace with parity $\left|v_{i}\right|=|i|$. The superalgebra $\operatorname{End}(\mathbf{V})$ has a basis formed of elementary matrices $E_{i j}: v_{k} \mapsto \delta_{j k} v_{i}$. Note that $\left|E_{i j}\right|=|i|+|j|$ and $E_{i j} E_{k l}=\delta_{j k} E_{i l}$. Recall the Perk-Schultz matrix from [27]:

$$
\begin{aligned}
R(z, w):= & \sum_{i \in I}\left(z q_{i}-w q_{i}^{-1}\right) E_{i i} \otimes E_{i i}+(z-w) \sum_{i \neq j} E_{i i} \otimes E_{j j} \\
& +z \sum_{i<j}\left(q_{i}-q_{i}^{-1}\right) E_{j i} \otimes E_{i j}+w \sum_{i<j}\left(q_{j}-q_{j}^{-1}\right) E_{i j} \otimes E_{j i} .
\end{aligned}
$$

Definition 3.1. [34] The quantum affine superalgebra $U_{q}(\widehat{\mathfrak{g}})$ is defined by

(R1) generators $s_{i j}^{(n)}, t_{i j}^{(n)}$ for $i, j \in I$ and $n \in \mathbb{Z}_{\geq 0}$;

(R2) parity $\left|s_{i j}^{(n)}\right|=\left|t_{i j}^{(n)}\right|=|i|+|j|$;

(R3) RTT-relations $[11,28]$ in $U_{q}(\widehat{\mathfrak{g}}) \otimes \operatorname{End}\left(\mathbf{V}^{\otimes 2}\right)\left[\left[z, z^{-1}, w, w^{-1}\right]\right]$ :

$$
\begin{aligned}
& R_{23}(z, w) T_{12}(z) T_{13}(w)=T_{13}(w) T_{12}(z) R_{23}(z, w), \\
& R_{23}(z, w) S_{12}(z) S_{13}(w)=S_{13}(w) S_{12}(z) R_{23}(z, w), \\
& R_{23}(z, w) T_{12}(z) S_{13}(w)=S_{13}(w) T_{12}(z) R_{23}(z, w), \\
& t_{i j}^{(0)}=s_{j i}^{(0)}=0 \quad \text { for } \quad 1 \leq i<j \leq M+N, \\
& t_{i i}^{(0)} s_{i i}^{(0)}=1=s_{i i}^{(0)} t_{i i}^{(0)} \quad \text { for } \quad i \in I .
\end{aligned}
$$

Here

$$
\begin{aligned}
& T(z)=\sum_{i, j \in I} t_{i j}(z) \otimes E_{i j} \in\left(U_{q}(\widehat{\mathfrak{g}}) \otimes \text { End } \mathbf{V}\right)\left[\left[z^{-1}\right]\right], \\
& t_{i j}(z)=\sum_{n \in \mathbb{Z}_{\geq 0}} t_{i j}^{(n)} z^{-n} \in U_{q}(\widehat{\mathfrak{g}})\left[\left[z^{-1}\right]\right]
\end{aligned}
$$

(similar convention for $S(z)$ with the $z^{-n}$ replaced by the $z^{n}$ ). 
The $q$-Yangian $Y_{q}(\mathfrak{g})$ is the subalgebra of $U_{q}(\widehat{\mathfrak{g}})$ generated by the $s_{i j}^{(n)},\left(s_{i i}^{(0)}\right)^{-1}$.

The quantum supergroup $U_{q}(\mathfrak{g})$ is the subalgebra of $U_{q}(\widehat{\mathfrak{g}})$ generated by the $s_{i j}^{(0)}$ and $t_{i j}^{(0)}$. We write $s_{i j}^{(0)}, t_{i j}^{(0)}$ as $s_{i j}, t_{i j}$ when no confusion with the series $s_{i j}(z), t_{i j}(z)$ arises.

Usual convention: if $A, B, C$ are superalgebras and $T=\sum_{i} a_{i} \otimes b_{i} \in A \otimes B$, then we write $T_{12}:=\sum_{i} a_{i} \otimes b_{i} \otimes 1 \in A \otimes B \otimes C, T_{13}:=\sum_{i} a_{i} \otimes 1 \otimes b_{i} \in A \otimes C \otimes B$ and $T_{23}:=\sum_{i} 1 \otimes a_{i} \otimes b_{i} \in$ $C \otimes A \otimes B$.

$U_{q}(\widehat{\mathfrak{g}})$ has a Hopf superalgebra structure with counit $\varepsilon: U_{q}(\widehat{\mathfrak{g}}) \longrightarrow \mathbb{C}$ defined by $\varepsilon\left(s_{i j}^{(n)}\right)=$ $\varepsilon\left(t_{i j}^{(n)}\right)=\delta_{i j} \delta_{n 0}$, and coproduct $\Delta: U_{q}(\widehat{\mathfrak{g}}) \longrightarrow U_{q}(\widehat{\mathfrak{g}})^{\otimes 2}$ :

$$
\Delta\left(s_{i j}^{(n)}\right)=\sum_{m=0}^{n} \sum_{k \in I} \epsilon_{i j k} s_{i k}^{(m)} \otimes s_{k j}^{(n-m)}, \quad \Delta\left(t_{i j}^{(n)}\right)=\sum_{m=0}^{n} \sum_{k \in I} \epsilon_{i j k} t_{i k}^{(m)} \otimes t_{k j}^{(n-m)} .
$$

Here $\epsilon_{i j k}:=(-1)^{\left|E_{i k}\right|\left|E_{k j}\right|}$. The antipode $\mathbb{S}: U_{q}(\widehat{\mathfrak{g}}) \longrightarrow U_{q}(\widehat{\mathfrak{g}})$ is determined by

$$
(\mathbb{S} \otimes \operatorname{Id})(S(z))=S(z)^{-1}, \quad(\mathbb{S} \otimes \operatorname{Id})(T(z))=T(z)^{-1} .
$$

Notice that $Y_{q}(\mathfrak{g})$ and $U_{q}(\mathfrak{g})$ are sub-Hopf-superalgebras of $U_{q}(\widehat{\mathfrak{g}})$.

We need the Drinfeld-Jimbo generators $e_{i}^{ \pm}, K_{i} \in U_{q}(\mathfrak{g})$ for $i \in I_{0}$ [34, Proposition 3.3]:

$$
e_{i}^{+}:=\frac{s_{i i}^{-1} s_{i, i+1}}{1-q_{i}^{-2}}, \quad e_{i}^{-}:=\frac{t_{i+1, i} t_{i i}^{-1}}{1-q_{i}^{2}}, \quad K_{i}:=s_{i i} s_{i+1, i+1}^{-1} .
$$

Let us recall the relations of these generators from [34]: ${ }^{4}$

$$
\begin{array}{ll}
{\left[e_{i}^{+}, e_{j}^{-}\right]=\delta_{i j} \frac{K_{i}-K_{i}^{-1}}{q_{i}-q_{i}^{-1}} \quad \text { for } \quad i, j \in I_{0},} \\
{\left[t_{j i}, t_{k j}\right]=\left(q_{j}-q_{j}^{-1}\right) t_{j j} t_{k i}, \quad\left[s_{i j}, s_{j k}\right]=\left(q_{j}-q_{j}^{-1}\right) s_{j j} s_{i k} \quad \text { if } \quad i<j<k,} \\
s_{i i}^{(0)} s_{j k}^{(n)}=q^{\left(\epsilon_{i}, \epsilon_{j}-\epsilon_{k}\right)} s_{j k}^{(n)} s_{i i}^{(0)}, & s_{i i}^{(0)} t_{j k}^{(n)}=q^{\left(\epsilon_{i}, \epsilon_{j}-\epsilon_{k}\right)} t_{j k}^{(n)} s_{i i}^{(0)} .
\end{array}
$$

Set $\mathfrak{g}^{\prime}:=\mathfrak{g l}(N, M)$. Let us define the quantum affine superalgebra $U_{q}\left(\widehat{\mathfrak{g}^{\prime}}\right)$ in the same way as $U_{q}(\widehat{\mathfrak{g}})$, except that $M, N$ are interchanged. Let $s_{i j}^{(n)}, t_{i j}^{\prime(n)}$ for $i, j \in I$ and $n \in \mathbb{Z}_{\geq 0}$ be the corresponding RTT generators of $U_{q}\left(\widehat{\mathfrak{g}}^{\prime}\right)$, so that their parities are $\left|s_{i j}^{\prime(n)}\right|=\left|t_{i j}^{(n)}\right|=|i|^{\prime}+|j|^{\prime}$ where $|i|^{\prime}=\overline{0}$ for $1 \leq i \leq N$ and $\overline{1}$ otherwise. For $i, j \in I$, set

$$
\varepsilon_{i j}:=(-1)^{|i|(|i|+|j|)}, \quad \varepsilon_{i j}^{\prime}:=(-1)^{|i|^{\prime}\left(|i|^{\prime}+|j|^{\prime}\right)}, \quad \widehat{i}:=M+N+1-i .
$$

Proposition 3.2. The following assignments define morphisms of superalgebras:

$$
\begin{aligned}
& \mathrm{ev}_{a}: U_{q}(\widehat{\mathfrak{g}}) \longrightarrow U_{q}(\mathfrak{g}), \quad s_{i j}(z) \mapsto s_{i j}-z a t_{i j}, \quad t_{i j}(z) \mapsto t_{i j}-z^{-1} a^{-1} s_{i j} ; \\
& \phi_{(f(z), g(z))}: U_{q}(\widehat{\mathfrak{g}}) \longrightarrow U_{q}(\widehat{\mathfrak{g}}), \quad s_{i j}(z) \mapsto f(z) s_{i j}(z), \quad t_{i j}(z) \mapsto g(z) t_{i j}(z) ; \\
& \Phi_{a}: \quad U_{q}(\widehat{\mathfrak{g}}) \longrightarrow U_{q}(\widehat{\mathfrak{g}}), \quad s_{i j}^{(n)} \mapsto a^{n} s_{i j}^{(n)}, \quad t_{i j}^{(n)} \mapsto a^{-n} t_{i j}^{(n)} ; \\
& \Psi: \quad U_{q}(\widehat{\mathfrak{g}}) \longrightarrow U_{q}(\widehat{\mathfrak{g}})^{\mathrm{cop}}, \quad s_{i j}^{(n)} \mapsto \varepsilon_{j i} t_{j i}^{(n)}, \quad t_{i j}^{(n)} \mapsto \varepsilon_{j i} s_{j i}^{(n)} ; \\
& \mathcal{F}: \quad U_{q}\left(\widehat{\mathfrak{g}^{\prime}}\right) \longrightarrow U_{q}(\widehat{\mathfrak{g}})^{\mathrm{cop}}, \quad s_{i j}^{\prime(n)} \mapsto \varepsilon_{j i}^{\prime} s_{\widetilde{j i}}^{(n)}, \quad t_{i j}^{\prime(n)} \mapsto \varepsilon_{j i}^{\prime} t_{\widetilde{j i}}^{(n)} .
\end{aligned}
$$

Here $a \in \mathbb{C}^{\times}$and $f(z), g\left(z^{-1}\right) \in 1+z \mathbb{C}[[z]]$. The last three maps are Hopf superalgebra isomorphisms. For $(A, \Delta, \varepsilon)$ a Hopf superalgebra, $\left(A^{\mathrm{cop}}, \Delta^{\mathrm{cop}}, \varepsilon\right)$ denotes another Hopf superalgebra with the same underlying superalgebra $A$ but with twisted coproduct $\Delta^{\text {cop }}:=c_{A, A} \Delta$, where $c_{A, A}: A^{\otimes 2} \longrightarrow A^{\otimes 2}$ is the graded permutation $x \otimes y \mapsto(-1)^{|x||y|} y \otimes x$.

\footnotetext{
${ }^{4}$ We use the super bracket $[x, y]:=x y-(-1)^{|x||y|} y x$. Equation (3.4) is [34, equation (A.20)] applied to by an evaluation map in Proposition 3.2 below. $\mathfrak{g}^{\prime}$ is not to be confused with the derived algebra of $\mathfrak{g}$.
} 
$\mathrm{ev}_{a}$ is called an evaluation map as ev $\circ \iota=\operatorname{Id}_{U_{q}(\mathfrak{g})}$. The maps $\phi_{(f(z), g(z))}, \Phi_{a}, \mathcal{F}$ restrict to $q$-Yangians. Relation (3.5) gives rise to the weight grading on $U_{q}(\widehat{\mathfrak{g}})$ : for $\alpha \in \mathbf{Q}$,

$$
U_{q}(\widehat{\mathfrak{g}})_{\alpha}=\left\{x \in U_{q}(\widehat{\mathfrak{g}}) \mid s_{i i}^{(0)} x\left(s_{i i}^{(0)}\right)^{-1}=q^{\left(\epsilon_{i}, \alpha\right)} x \text { for } i \in I\right\} .
$$

For example $s_{i j}^{(n)}, t_{i j}^{(n)}$ are of weight $\epsilon_{i}-\epsilon_{j}$. This induces weight gradings on $Y_{q}(\mathfrak{g})$ and $U_{q}(\mathfrak{g})$.

We end this section with some facts on representations of $U_{q}(\mathfrak{g})$ following [9].

A $U_{q}(\mathfrak{g})$-module $V$ admits a weight grading if it is a direct sum of weight spaces

$$
V_{\alpha}:=\left\{x \in V \mid s_{i i} x=q^{\left(\epsilon_{i}, \alpha\right)} x \text { for } i \in I\right\} \quad \text { with } \quad \alpha \in \mathbf{P} .
$$

By equation (3.5), $U_{q}(\mathfrak{g})_{\alpha} V_{\beta} \subseteq V_{\alpha+\beta}$ for $\alpha, \beta \in \mathbf{P}$. If furthermore all the weight spaces $V_{\alpha}$ are finite-dimensional, then the character of $V$ can be defined:

$$
\chi(V):=\sum_{\alpha \in \mathbf{P}} \operatorname{dim}(V)_{\alpha}[\alpha] \in \mathbb{Z}^{\mathbf{P}} .
$$

Here $\mathbb{Z}^{\mathbf{P}}$ is the abelian group of functions $\mathbf{P} \longrightarrow \mathbb{Z}$ and $[\alpha]: \beta \mapsto \delta_{\alpha, \beta}$.

Let $\lambda \in \mathbf{P}$. Up to isomorphism, there exists a unique irreducible $U_{q}(\mathfrak{g})$-module, denoted by $L(\lambda)$, which is generated by a vector $v_{\lambda}$ satisfying

$$
\left|v_{\lambda}\right|=|\lambda|, \quad s_{k k} v_{\lambda}=q^{\left(\epsilon_{k}, \lambda\right)} v_{\lambda}, \quad t_{k k} v_{\lambda}=q^{-\left(\epsilon_{k}, \lambda\right)} v_{\lambda}, \quad s_{i j} v_{\lambda}=0, \quad i, j, k \in I, \quad i<j .
$$

$L(\lambda)$ is weight graded with finite-dimensional weight spaces. $L(\lambda)_{\lambda}=\mathbb{C} v_{\lambda}$ and $L(\lambda)_{\alpha} \neq 0$ only if $\lambda-\alpha \in \mathbf{Q}_{+}$. (The proof, parallel to the non-graded case, is based on the triangular decomposition and PBW basis of $U_{q}(\mathfrak{g})$ in [32].)

The first example is the vector representation $\pi$ of $U_{q}(\mathfrak{g})$ on $\mathbf{V}$ [34, Example 1]:

$$
\begin{aligned}
& \pi\left(s_{i i}\right)=q_{i} E_{i i}+\sum_{j \neq i} E_{j j}=\pi\left(t_{i i}^{-1}\right) \quad \text { for } \quad i \in I, \\
& \pi\left(s_{i j}\right)=\left(q_{i}-q_{i}^{-1}\right) E_{i j}, \quad \pi\left(t_{j i}\right)=\left(q_{i}^{-1}-q_{i}\right) E_{j i} \quad \text { for } \quad 1 \leq i<j \leq M+N .
\end{aligned}
$$

We have $(\pi, \mathbf{V}) \cong L\left(\epsilon_{1}\right)$ with $v_{1}=v_{\epsilon_{1}}$ and $\chi\left(L\left(\epsilon_{1}\right)\right)=\sum_{i \in I}\left[\epsilon_{i}\right]$.

Definition 3.3. [34] Kirillov-Reshetikhin module $W_{k, a}^{(r)}$ for $r \in I_{0}, k \in \mathbb{Z}_{>0}, a \in \mathbb{C}^{\times}$is

(1) either $\mathrm{ev}_{a q^{2 k}}^{*} L\left(k \varpi_{r}\right)$ with $\varpi_{r}=\sum_{j=1}^{r} \epsilon_{j}$ and $r \leq M$,

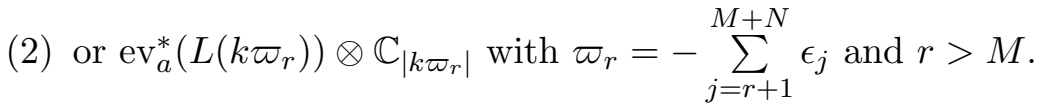

Here $\mathbb{C}_{s}$ for $s \in \mathbb{Z}_{2}$ is the one-dimensional $U_{q}(\widehat{\mathfrak{g}})$-module $\varepsilon: U_{q}(\widehat{\mathfrak{g}}) \longrightarrow \mathbb{C}$ of parity $s$

Let $r, k \in \mathbb{Z}_{>0}$ be such that $r \leq M$ or $k \leq N$. Consider the rectangle Young diagram $Y_{r, k}$ with $r$ rows and $k$ columns. We view $Y_{r, k}$ as a subset of $\mathbb{Z}_{>0} \times \mathbb{Z}_{>0}$ so that $(i, j) \in Y_{r, k}$ corresponds to the box at $i$-th row and $j$-th column. An $(M, N)$-hook semi-standard tableau of shape $Y_{r, k}$ is a function $T: Y_{r, k} \longrightarrow I=\{1<2<\cdots<M+N\}$ such that:

(i) the entries in each row and column are weakly increasing;

(ii) the entries in $\{1,2, \ldots, M\}$ are strictly increasing in each column;

(iii) the entries in $\{M+1, M+2, \ldots, M+N\}$ are strictly increasing in each row; 
Let $\mathcal{B}_{r, k}$ be the set of all such functions.

Theorem 3.4 ([9]). For $1 \leq r \leq M$ and $k \in \mathbb{Z}_{>0}$

$$
\chi\left(L\left(k \varpi_{r}\right)\right)=\sum_{T \in \mathcal{B}_{r, k}}\left[\sum_{(i, j) \in Y_{r, k}} \epsilon_{T(i, j)}\right] .
$$

If $r=M$ and $k \geq N$, then $\operatorname{dim} L\left(k \varpi_{M}\right)=2^{M N}$.

As a consequence, $L\left(k \varpi_{r}\right)$ is finite-dimensional for $1 \leq r \leq M$. This is also true for $M<$ $r \leq M+N$. Indeed, the pullback of the $U_{q}(\mathfrak{g})$-module $L\left(k \varpi_{r}\right)$ by $\mathcal{F}: U_{q}\left(\mathfrak{g}^{\prime}\right) \longrightarrow U_{q}(\mathfrak{g})$ in Proposition 3.2 is an irreducible module over $U_{q}\left(\mathfrak{g}^{\prime}\right)$ of highest weight $k \varpi_{M+N-r}^{\prime}$ (we add prime to distinguish $\mathfrak{g}^{\prime}=\mathfrak{g l}(N, M)$ with $\left.\mathfrak{g}\right)$ so that its character can be computed by equation (3.8) in terms of $(N, M)$-hook semi-standard tableaux of shape $Y_{M+N-r, k}$.

In [9], the tableaux correspond to Kashiwara's crystal basis of $L(\lambda)$. As an example: $\mathfrak{g}=$

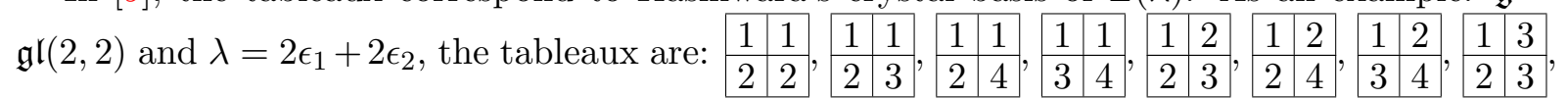

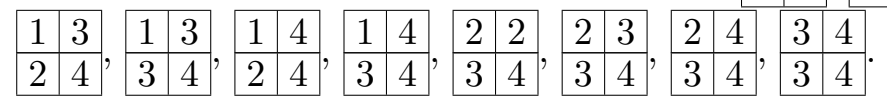

\section{Inductive system of Kirillov-Reshetikhin modules}

We construct inductive system of KR modules, based on our previous result [34] and establish its asymptotic property as $U_{q}(\mathfrak{g})$-modules.

Let $V$ be a $U_{q}(\widehat{\mathfrak{g}})$-module. A $\mathbb{Z}_{2}$-homogeneous non-zero vector $v \in V$ is called a highest $\ell$ weight vector if it is a common eigenvector for the $s_{i i}^{(n)}, t_{i i}^{(n)}$ and it is annihilated by the $s_{i j}^{(n)}, t_{i j}^{(n)}$ with $i<j . \quad V$ is called a highest $\ell$-weight module if $V=U_{q}(\widehat{\mathfrak{g}}) v$ for some highest $\ell$-weight vector $v$. In this case, $v$ is unique up to scalar multiple, and $V$ admits a unique irreducible quotient, called the head of $V$ and denoted by $\mathrm{hd}(V)$.

For example, let $v_{\lambda} \in L(\lambda)$ be as in Section 3 , and let $a \in \mathbb{C}^{\times}$. The evaluation module $\mathrm{ev}_{a}^{*} L(\lambda)$ contains a highest $\ell$-weight vector $w:=\operatorname{ev}_{a}^{*}\left(v_{\lambda}\right)$ with

$$
|w|=|\lambda|, \quad s_{i i}(z) w=\left(q^{\left(\epsilon_{i}, \lambda\right)}-z a q^{-\left(\epsilon_{i}, \lambda\right)}\right) w, \quad t_{i i}(z) w=\left(q^{-\left(\epsilon_{i}, \lambda\right)}-z^{-1} a^{-1} q^{\left(\epsilon_{i}, \lambda\right)}\right) w .
$$

The tensor product of two highest $\ell$-weight vectors is also a highest $\ell$-weight vector. Let $V, V^{\prime}$ be $U_{q}(\widehat{\mathfrak{g}})$-modules. We write $V \simeq V^{\prime}$ if there exists a one-dimensional $U_{q}(\widehat{\mathfrak{g}})$-module $D$ such that $V \cong V^{\prime} \otimes D$ as $U_{q}(\widehat{\mathfrak{g}})$-modules. In this case, since $\left.s_{i j}^{(n)}\right|_{D}=\left.t_{i j}^{(n)}\right|_{D}=0$ for $i \neq j$, we have that $V$ is of highest $\ell$-weight if and only if so is $V^{\prime}$.

Theorem 4.1 ([34, Theorem 5.2]). Let $r \in I_{0}$ and $a_{1}, a_{2}, \ldots, a_{k} \in \mathbb{C}^{\times}$. The $U_{q}(\widehat{\mathfrak{g}})$-module $W_{1, a_{1}}^{(r)} \otimes W_{1, a_{2}}^{(r)} \otimes \cdots \otimes W_{1, a_{k}}^{(r)}$ is of highest $\ell$-weight if $\frac{a_{i}}{a_{j}} \notin q_{r}^{\mathbb{Z}^{<0}}$ for $1 \leq i<j \leq k .^{5}$

If a sequence $\left(X_{j}\right)_{j \in \mathbb{Z}}$ of $Y_{q}(\mathfrak{g})$-modules is given and $m, n \in \mathbb{Z}$ with $m \leq n$, then we write

$$
X_{n} \otimes X_{n-1} \otimes \cdots \otimes X_{m+1} \otimes X_{m}=: \bigotimes_{m \leq j \leq n}^{\overleftarrow{ }} X_{j}
$$

For example, $\bigotimes_{0 \leq j \leq k-1}^{\overleftarrow{\bigotimes}} W_{1, a q_{r}^{2 j}}^{(r)}$ is of highest $\ell$-weight for $k \in \mathbb{Z}_{>0}$

\footnotetext{
${ }^{5}$ This result has been made stronger in the author's later works $[35,36]$.
} 
Lemma 4.2. Let $r \in I_{0}, a \in \mathbb{C}^{\times}$and $k \in \mathbb{Z}_{>0}$. Then $\operatorname{hd}\left(\underset{0 \leq j \leq k-1}{\overleftarrow{\bigotimes}} W_{1, a q_{r}^{2 j}}^{(r)}\right) \simeq W_{k, a}^{(r)}$

Proof. Let us compare the highest $\ell$-weight vectors $v, v^{\prime}$ at the left-hand and right-hand sides respectively. Write $s_{i i}(z) v=f_{i}(z) v$ and $s_{i i}(z) v^{\prime}=f_{i}^{\prime}(z) v^{\prime}$ for $i \in I$. By Definition 3.3 and Proposition 3.2, $f_{i}(z)=f_{i+1}(z)$ and $f_{i}^{\prime}(z)=f_{i+1}^{\prime}(z)$ for $i \in I_{0} \backslash\{r\}$,

$$
\frac{f_{r}(z)}{f_{r+1}(z)}=\prod_{j=1}^{k} \frac{1-z a q_{r}^{2 k-2 j}}{q_{r}^{-1}-z a q_{r}^{2 k-2 j+1}}=\frac{1-z a}{q_{r}^{-k}-z a q_{r}^{k}}=\frac{f_{r}^{\prime}(z)}{f_{r+1}^{\prime}(z)}
$$

Similar statement holds for eigenvalues of the $t_{i i}(z)$. Set $g^{ \pm}=\prod_{j=1}^{k-1}\left(1-z^{ \pm 1} a^{ \pm 1} q_{r}^{ \pm 2 j}\right)$. Then $\operatorname{hd}\left(\overleftarrow{\bigotimes}_{0 \leq j \leq k-1}^{\overleftarrow{ }} W_{1, a q_{r}^{2 j}}^{(r)}\right) \cong W_{k, a}^{(r)} \otimes \phi_{\left(g^{+}, g^{-}\right)}^{*}\left(\mathbb{C}_{\overline{0}}\right) ;$ see Definition 3.3(2)

From now on up to the end of Section $5, r \in I_{0}$ and $a \in \mathbb{C}^{\times}$are fixed.

For $k \in \mathbb{Z}_{>0}$, let $\rho^{k}$ denote the representation of $U_{q}(\widehat{\mathfrak{g}})$ on $W_{k, a}^{(r)}$ and let us fix a highest $\ell$-weight vector $\omega_{k}$ in $W_{k, a}^{(r)}$. If $l, k \in \mathbb{Z}_{>0}$ and $l<k$, then define the $U_{q}(\widehat{\mathfrak{g}})$-module

$$
Z_{k l}:=\phi_{\left(\left(1-z a q_{r}^{2 l}\right)^{-1},\left(1-z^{-1} a^{-1} q_{r}^{-2 l}\right)^{-1}\right)}^{*} W_{k-l, a q_{r}^{2 l}}^{(r)},
$$

and fix a highest $\ell$-weight vector $\omega_{k l}$ in $Z_{k l}$. Let us show that $Z_{k l} \otimes W_{l, a}^{(r)}$ is of highest $\ell$ weight. By Lemma 4.2, one may replace $Z_{k l}$ and $W_{l, a}^{(r)}$ by the heads of the tensor products

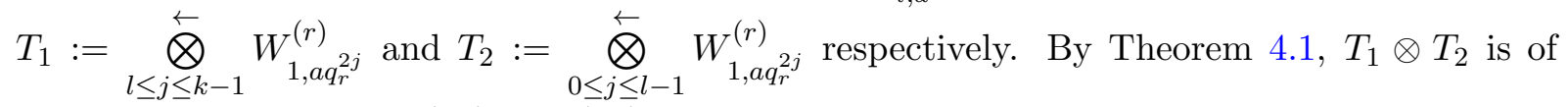
highest $\ell$-weight, so is $\mathrm{hd}\left(T_{1}\right) \otimes \mathrm{hd}\left(T_{2}\right)$ as its quotient.

From the proof of Lemma 4.2 follows $h d\left(Z_{k l} \otimes W_{l, a}^{(r)}\right) \cong W_{k, a}^{(r)}$. As in [21, Section 4], let $\mathscr{F}_{k, l}: Z_{k l} \otimes W_{l, a}^{(r)} \longrightarrow W_{k, a}^{(r)}$ be the quotient map sending $\omega_{k l} \otimes \omega_{l}$ to $\omega_{k}$, and define the restriction $\operatorname{map} F_{k, l}: W_{l, a}^{(r)} \longrightarrow W_{k, a}^{(r)}, x \mapsto \mathscr{F}_{k, l}\left(\omega_{k l} \otimes x\right)$. In particular $F_{k, l}\left(\omega_{l}\right)=\omega_{k}$.

Proposition 4.3. The maps $\left(F_{k, l}: W_{l, a}^{(r)} \longrightarrow W_{k, a}^{(r)}\right)_{l<k}$ verify the following properties.

(1) $F_{k, l}\left(W_{l, a}^{(r)}\right)_{l \varpi_{r}-\beta} \subseteq\left(W_{k, a}^{(r)}\right)_{k \varpi_{r}-\beta}$ for $\beta \in \mathbf{Q}_{+}$, and $\rho^{k}\left(e_{i}^{+}\right) F_{k, l}=F_{k, l} \rho^{l}\left(e_{i}^{+}\right)$for $i \in I_{0}$.

(2) $F_{k, l}: W_{l, a}^{(r)} \longrightarrow W_{k, a}^{(r)}$ is injective, and $F_{k, l} F_{l, m}=F_{k, m}$ for $m<l<k$.

(3) $\rho^{k}\left(e_{i}^{-}\right) F_{k, l}=F_{k, l} \rho^{l}\left(e_{i}^{-}\right)$for $i \in I_{0} \backslash\{r\}$. For $m>0$, there exist linear maps $A_{m}, B_{m}: W_{m, a}^{(r)}$ $\longrightarrow W_{m+1, a}^{(r)}$ of parity $\left|\alpha_{r}\right|$ such that

$$
\rho^{k}\left(e_{r}^{-}\right) F_{k, m}=F_{k, m+1}\left(q_{r}^{k} A_{m}+q_{r}^{-k} B_{m}\right): W_{m, a}^{(r)} \longrightarrow W_{k, a}^{(r)} \quad \text { for } \quad k>m+1 .
$$

Proof. (1) By definition $\omega_{k l}$ is of weight $(k-l) \varpi_{r}$. Since the $U_{q}(\widehat{\mathfrak{g}})$-linear map $\mathscr{F}_{k, l}$ respects the weight gradings, $F_{k, l}$ changes the weights by $(k-l) \varpi_{r}$. By equation (3.2),

$$
\Delta\left(e_{i}^{+}\right)=1 \otimes e_{i}^{+}+e_{i}^{+} \otimes K_{i}^{-1}, \quad \Delta\left(e_{i}^{-}\right)=K_{i} \otimes e_{i}^{-}+e_{i}^{-} \otimes 1 .
$$

Since $e_{i}^{+} \omega_{k l}=0$, we have: for $x \in W_{l, a}^{(r)}$,

$$
e_{i}^{+} F_{k, l}(x)=e_{i}^{+} \mathscr{F}_{k, l}\left(\omega_{k l} \otimes x\right)=\mathscr{F}_{k, l}\left(\Delta\left(e_{i}^{+}\right)\left(\omega_{k l} \otimes x\right)\right)=\mathscr{F}_{k, l}\left(\omega_{k l} \otimes e_{i}^{+} x\right)=F_{k, l} e_{i}^{+}(x) .
$$


(2) Assume $\operatorname{ker}\left(F_{k, l}\right) \neq 0$. By (1) there exists $\mu \in \mathbf{P}$ such that $\operatorname{ker}\left(F_{k, l}\right)_{\mu+\alpha_{i}}=0$ and $\operatorname{ker}\left(F_{k, l}\right)_{\mu} \neq 0$ for $i \in I_{0}$. This implies $e_{i}^{+} \operatorname{ker}\left(F_{k, l}\right)_{\mu}=0$ for all $i \in I_{0}$. By equation (3.4), $s_{i j} \operatorname{ker}\left(F_{k, l}\right)_{\mu}=0$ for $1 \leq i<j \leq M+N$. Since $W_{l, a}^{(r)} \cong L\left(l \varpi_{r}\right) \otimes \mathbb{C}_{\left|l \varpi_{r}\right|}$ is an irreducible $U_{q}(\mathfrak{g})$-module, we have $\operatorname{ker}\left(F_{k, l}\right)_{\mu} \subseteq\left(W_{l, a}^{(r)}\right)_{l \varpi_{r}}=\mathbb{C} \omega_{l}$, in contradiction with $F_{k, l}\left(\omega_{l}\right)=\omega_{k}$.

Consider the $U_{q}(\widehat{\mathfrak{g}})$-module $S:=Z_{k l} \otimes Z_{l m} \otimes W_{m, a}^{(r)}$. It contains a highest $\ell$-weight vector $\omega:=\omega_{k l} \otimes \omega_{l m} \otimes \omega_{m}$. By Lemma 4.2 we have as $U_{q}(\widehat{\mathfrak{g}})$-modules

$$
S \simeq\left(\bigotimes_{l \leq j \leq k-1}^{\overleftarrow{\bigotimes}} W_{1, a q_{r}^{2 j}}^{(r)}\right) \otimes\left(\bigotimes_{m \leq j \leq l-1}^{\overleftarrow{\bigotimes}} W_{1, a q_{r}^{2 j}}^{(r)}\right) \otimes\left(\bigotimes_{0 \leq j \leq m-1}^{\overleftarrow{\bigotimes}} W_{1, a q_{r}^{2 j}}^{(r)}\right)
$$

Theorem 4.1 applied to the right-hand side, $S$ and $Z_{k l} \otimes Z_{l m}$ are of highest $\ell$-weight with heads $W_{k, a}^{(r)}$ and $Z_{k m}$ respectively. Let $\mathscr{G}_{k, m}^{l}: Z_{k l} \otimes Z_{l m} \longrightarrow Z_{k m}$ be the quotient map sending $\omega_{k l} \otimes$ $\omega_{l m}$ to $\omega_{k m}$. We obtain $U_{q}(\widehat{\mathfrak{g}})$-module morphisms from $S$ to $W_{k, a}^{(r)}$ :

$$
F:=\mathscr{F}_{k, l}\left(\operatorname{Id}_{Z_{k l}} \otimes \mathscr{F}_{l, m}\right), \quad G:=\mathscr{F}_{k, m}\left(\mathscr{G}_{k, m}^{l} \otimes \operatorname{Id}_{W_{l, a}^{(r)}}\right)
$$

Since $F(\omega)=G(\omega)=\omega_{k}$ and since $S$ is generated by $\omega$, we have $F=G$. Applying $F, G$ to $\omega_{k l} \otimes \omega_{l m} \otimes W_{m, a}^{(r)}$ gives the desired identity of (2).

(3) Assume $i \in I_{0} \backslash\{r\}$. Since $e_{i}^{+} \omega_{l}=0, K_{i} \omega_{l}=\omega_{l}$, by (3.3), $e_{i}^{+} e_{i}^{-} \omega_{l}=0$. If $j \in I_{0} \backslash\{i\}$, then $e_{j}^{+} e_{i}^{-} \omega_{l} \in W_{l, a}^{(r)}$ is of weight $l \varpi_{r}-\alpha_{i}+\alpha_{j} \notin l \varpi_{r}+\mathbf{Q}_{-}$and is zero. So $e_{i}^{-} \omega_{l}$ is annihilated by all the $e_{j}^{+}$. As in (2), $e_{i}^{-} \omega_{l}=0$. Then $e_{i}^{-} F_{k, l}=F_{k, l} e_{i}^{-}$as in (1), using $\Delta\left(e_{i}^{-}\right)$.

For $i=r$, we adapt the proof of [36, Lemma 7.6]. We fix $l=m+1$ in (2). Applying $F=G$ to $\omega_{k l} \otimes w^{\prime} \otimes w \in S$ for $w^{\prime} \in Z_{l m}$ and $w \in W_{m, a}^{(r)}$ and $k>l$ gives the identity:

$$
F_{k, l} \mathscr{F}_{l, m}\left(w^{\prime} \otimes w\right)=\mathscr{F}_{k, m}\left(\mathscr{G}_{k, m}^{l}\left(\omega_{k l} \otimes w^{\prime}\right) \otimes w\right) .
$$

We compute $e_{r}^{-} \omega_{k m}$ via the projection $\mathscr{G}_{k m}^{l}\left(\omega_{k l} \otimes \omega_{l m}\right)=\omega_{k m}$ and $K_{r} \omega_{k l}=q_{r}^{k-l} \omega_{k l}$

$$
e_{r}^{-} \omega_{k m}=\mathscr{G}_{k, m}^{l}\left(q_{r}^{k-l} \omega_{k l} \otimes e_{r}^{-} \omega_{l m}+e_{r}^{-} \omega_{k l} \otimes \omega_{l m}\right) .
$$

Next consider the following vector in $Z_{k l} \otimes Z_{l m}$ of weight $(k-m) \varpi_{r}-\alpha_{r}$ :

$$
x:=q_{r}^{-1} \frac{q_{r}^{k-l}-q_{r}^{l-k}}{q_{r}-q_{r}^{-1}} \omega_{k l} \otimes e_{r}^{-} \omega_{l m}-e_{r}^{-} \omega_{k l} \otimes \omega_{l m} .
$$

Based on $\Delta\left(e_{r}^{+}\right)$and $l=m+1$ one checks that $e_{r}^{+} x=0$. If $j \in I_{0} \backslash\{r\}$, then $e_{j}^{+} x$ is of weight $(k-m) \varpi_{r}-\alpha_{r}+\alpha_{j} \notin(k-m) \varpi_{r}+\mathbf{Q}_{-}$and is zero. So $\mathscr{G}_{k, m}^{l}(x) \in Z_{k m}$ is annihilated by the $e_{j}^{+}$ for $j \in I_{0}$. As in (2), $\mathscr{G}_{k, m}^{l}(x)=0$. It follows that

$$
e_{r}^{-} \omega_{k m}=\mathscr{G}_{k, m}^{l}\left(q_{r}^{k-l} \omega_{k l} \otimes e_{r}^{-} \omega_{l m}+e_{r}^{-} \omega_{k l} \otimes \omega_{l m}+x\right)=\frac{q_{r}^{k-m}-q_{r}^{m-k}}{q_{r}-q_{r}^{-1}} \mathscr{G}_{k, m}^{l}\left(\omega_{k l} \otimes e_{r}^{-} \omega_{l m}\right) .
$$

Taking $w^{\prime}:=e_{r}^{-} \omega_{l m} \in Z_{l m}$ in (4.1), we compute

$$
\begin{aligned}
& e_{r}^{-} F_{k, m}(w)=e_{r}^{-} \mathscr{F}_{k, m}\left(\omega_{k m} \otimes w\right)=\mathscr{F}_{k, m}\left(e_{r}^{-} \omega_{k m} \otimes w\right)+q_{r}^{k-m} \mathscr{F}_{k, m}\left(\omega_{k m} \otimes e_{r}^{-} w\right) \\
& =\frac{q_{r}^{k-m}-q_{r}^{m-k}}{q_{r}-q_{r}^{-1}} \mathscr{F}_{k, m}\left(\mathscr{G}_{k, m}^{l}\left(\omega_{k l} \otimes e_{r}^{-} \omega_{l m}\right) \otimes w\right)+q_{r}^{k-m} F_{k, m}\left(e_{r}^{-} w\right) \\
& =\frac{q_{r}^{k-m}-q_{r}^{m-k}}{q_{r}-q_{r}^{-1}} F_{k, l} \mathscr{F}_{l, m}\left(e_{r}^{-} \omega_{l m} \otimes w\right)+q_{r}^{k-m} F_{k, m}\left(e_{r}^{-} w\right) .
\end{aligned}
$$


This shows that $\rho^{k}\left(e_{r}^{-}\right) F_{k, m}=F_{k, m+1}\left(q_{r}^{k} A_{m}+q_{r}^{-k} B_{m}\right)$ where

$$
\begin{aligned}
& A_{m}(w):=q_{r}^{-m} F_{m+1, m}\left(e_{r}^{-} w\right)+\frac{q_{r}^{-m}}{q_{r}-q_{r}^{-1}} \mathscr{F}_{m+1, m}\left(e_{r}^{-} \omega_{m+1, m} \otimes w\right), \\
& B_{m}(w):=\frac{-q_{r}^{m}}{q_{r}-q_{r}^{-1}} \mathscr{F}_{m+1, m}\left(e_{r}^{-} \omega_{m+1, m} \otimes w\right),
\end{aligned}
$$

as linear maps $W_{m, a}^{(r)} \longrightarrow W_{m+1, a}^{(r)}$ are clearly independent of $k$ and of parity $\left|\alpha_{r}\right|$.

Remark 4.4. By equation (3.4), for $1 \leq i<j \leq M+N, s_{i i}^{-1} s_{i j}$ (resp. $t_{j i} t_{i i}^{-1}$ ) is a sum of monomials of the $e_{i}^{+}, e_{i+1}^{+}, \ldots, e_{j-1}^{+}$(resp. the $e_{i}^{-}, e_{i+1}^{-}, \ldots, e_{j-1}^{-}$) where each $e_{h}^{ \pm}$for $i \leq h<j$ appears once. It follows that $\rho^{k}\left(s_{i i}^{-1} s_{i j}\right) F_{k, l}=F_{k, l} \rho^{l}\left(s_{i i}^{-1} s_{i j}\right)$ and:

(i) if $i>r$ or $j \leq r$, then $\rho^{k}\left(t_{j i} t_{i i}^{-1}\right) F_{k, l}=F_{k, l} \rho^{l}\left(t_{j i} t_{i i}^{-1}\right)$ for $k>l$;

(ii) if $i \leq r<j$, then $\rho^{k}\left(t_{j i} t_{i i}^{-1}\right) F_{k, l}=F_{k, l+1}\left(q_{r}^{k} A_{j i ; l}+q_{r}^{-k} B_{j i ; l}\right)$ for $k>l+1$, where $A_{j i ; l}, B_{j i ; l}$ are linear maps $W_{l, a}^{(r)} \longrightarrow W_{l+1, a}^{(r)}$ of parity $\left|\epsilon_{j}-\epsilon_{i}\right|$.

For example, $A_{r+1, r ; l}=A_{l}$ and $B_{r+1, r ; l}=B_{l}$. Furthermore, for $i \in I$ and $l<k$ we have

$$
\rho^{k}\left(s_{i i}\right) F_{k, l}=q^{\left(\epsilon_{i},(k-l) \varpi_{r}\right)} F_{k, l} \rho^{l}\left(s_{i i}\right), \quad \rho^{k}\left(t_{i i}\right) F_{k, l}=q^{-\left(\epsilon_{i},(k-l) \varpi_{r}\right)} F_{k, l} \rho^{l}\left(t_{i i}\right) .
$$

\section{$5 \quad$ Asymptotic representations}

We apply the asymptotic constructions of Section $2\left(c \in \mathbb{C}^{\times}\right.$or $\left.c=0\right)$ to the inductive system of KR modules in Proposition 4.3, and obtain $U_{q}(\widehat{\mathfrak{g}})$ or $Y_{q}(\mathfrak{g})$-modules. Set $T:=\left\{s_{i j}^{(n)}, t_{i j}^{(n)}\right\}$ (resp. $\left.S:=\left\{s_{i j}^{(n)}\right\}\right)$ to be the system of algebraic generators of $U_{q}(\widehat{\mathfrak{g}})$ (resp. $Y_{q}(\mathfrak{g})$ ).

For $k>0$, the map $S \ni s_{i j}^{(n)} \mapsto q^{\left(\epsilon_{i},-k \varpi_{r}\right)} \rho^{k}\left(s_{i j}^{(n)}\right) \in \operatorname{End}\left(W_{k, a}^{(r)}\right)$ extends uniquely to a representation $\left(\tilde{\rho}^{k}, W_{k, a}^{(r)}\right)$ of $Y_{q}(\mathfrak{g})$. Indeed, there is a representation $\left(\theta_{k}^{(r)}, \mathbb{C}\right)$ of $Y_{q}(\mathfrak{g})$ on the one-dimensional vector superspace of even parity given by $\theta_{k}^{(r)}\left(s_{i j}^{(n)}\right)=q^{\left(\epsilon_{i},-k \varpi_{r}\right)} \delta_{i j} \delta_{n, 0}$ and $\left(\tilde{\rho}^{k}, W_{k, a}^{(r)}\right) \cong\left(\theta_{k}^{(r)}, \mathbb{C}\right) \otimes\left(\rho^{k}, W_{k, a}^{(r)}\right)$ as $Y_{q}(\mathfrak{g})$-modules. ${ }^{6}$

Lemma 5.1. Let $t \in T, s \in S$ and $l>0$. There exist Hom $\left(W_{l, a}^{(r)}, W_{l+1, a}^{(r)}\right)$-valued Laurent polynomials $P_{t ; l}(u)=\sum_{i=-2}^{2} P_{t ; l}^{[i]} u^{i}$ and $Q_{s ; l}(u)=\sum_{i=0}^{1} Q_{s ; l}^{[i]} u^{i}$ in $u$ of parity $|t|$ and $|s|$ respectively such that for all $k>l+1$ the following identities hold in $\operatorname{Hom}\left(W_{l, a}^{(r)}, W_{k, a}^{(r)}\right)$ :

$$
\rho^{k}(t) F_{k, l}=\left.F_{k, l+1} P_{t ; l}(u)\right|_{u=q_{r}^{k}}, \quad \tilde{\rho}^{k}(s) F_{k, l}=\left.F_{k, l+1} Q_{s ; l}(u)\right|_{u=q_{r}^{2 k}} .
$$

Proof. If $r \leq M$, then for $i, j \in I$ we have as power series in $z$ :

$$
\begin{aligned}
\sum_{n \geq 0} z^{n} \rho^{k}\left(s_{i j}^{(n)}\right) F_{k, l} & =\rho^{k}\left(s_{i j}\right) F_{k, l}-z a q^{2 k} \rho^{k}\left(t_{i j}\right) F_{k, l} \\
& =q^{(k-l)\left(\epsilon_{i}, \varpi_{r}\right)} F_{k, l} \rho^{l}\left(s_{i j}\right)-z a q^{2 k-(k-l)\left(\epsilon_{j}, \varpi_{r}\right)} \rho^{k}\left(t_{i j} t_{j j}^{-1}\right) F_{k, l} \rho^{l}\left(t_{j j}\right),
\end{aligned}
$$

and $\rho^{k}\left(t_{i j}^{(n)}\right)=-a^{-1} q^{-2 k} \rho^{k}\left(s_{i j}^{(1-n)}\right)$. If $r>M$, then $\rho^{k}\left(t_{i j}^{(n)}\right)=-a^{-1} \rho^{k}\left(s_{i j}^{(1-n)}\right)$ and

$$
\sum_{n \geq 0} z^{n} \rho^{k}\left(s_{i j}^{(n)}\right) F_{k, l}=q^{(k-l)\left(\epsilon_{i}, \varpi_{r}\right)} \rho^{k}\left(s_{i j}\right) F_{k, l}-z a q^{-(k-l)\left(\epsilon_{j}, \varpi_{r}\right)} \rho^{k}\left(t_{i j} t_{j j}^{-1}\right) F_{k, l} \rho^{l}\left(t_{j j}\right) .
$$

\footnotetext{
${ }^{6}$ The $\tilde{\rho}^{k}$ are normalized Kirillov-Reshetikhin modules in [14].
} 
We compute the right-hand sides in four cases based on Remark 4.4:

\begin{tabular}{|c|c|c|}
\hline$r \leq M$ & $r>M$ & \\
\hline$q^{k} \times F_{k, l} \rho^{l}\left(q^{-l} s_{i j}\right)$ & $F_{k, l} \rho^{l}\left(s_{i j}\right)$ & $i \leq r<j$ \\
\hline$F_{k, l} \rho^{l}\left(s_{i j}-z a q^{2 k} t_{i j}\right)$ & $q^{k} \times F_{k, l} \rho^{l}\left(q^{-l} s_{i j}-z a q^{-2 k+l} t_{i j}\right)$ & $r<i, j$ \\
\hline$-z a F_{k, l+1}\left(q^{2 k+l} A_{i j ; l}+q^{l} B_{i j ; l}\right)$ & $q^{k} \times(-z a) F_{k, l+1}\left(q^{-2 k} A_{i j ; l}+B_{i j ; l}\right)$ & $j \leq r<i$ \\
\hline$q^{k} \times F_{k, l} \rho^{l}\left(q^{-l} s_{i j}-z a q^{l} t_{i j}\right)$ & $F_{k, l} \rho^{l}\left(s_{i j}-z a t_{i j}\right)$ & $i, j \leq r$ \\
\hline
\end{tabular}

Removing the factors " $q^{k} \times$ " gives $\tilde{\rho}^{k}\left(s_{i j}(z)\right) F_{k, l}$, a polynomial in $q_{r}^{2 k}$ of degree $\leq 1$.

Let $\left(F_{l}: W_{l, a}^{(r)} \longrightarrow W_{\infty}\right)_{l>0}$ be the inductive limit of $\left(F_{k, l}: W_{l, a}^{(r)} \longrightarrow W_{k, a}^{(r)}\right)$.

Corollary 5.2. Fix $c \in \mathbb{C}^{\times}$. There exist representations $\rho_{c}$ of $U_{q}(\widehat{\mathfrak{g}})$ and $\rho_{+}$of $Y_{q}(\mathfrak{g})$ on $W_{\infty}$ defined by the formulas: for $t \in T, s \in S$,

$$
\rho_{c}(t)=\left.\lim _{l \rightarrow \infty} P_{t ; l}(u)\right|_{u=c}, \quad \rho_{+}(s)=\left.\lim _{l \rightarrow \infty} Q_{s ; l}(u)\right|_{u=0}
$$

Let $W_{a ; c}^{(r)}$ and $L_{r, a}^{+}$denote the $U_{q}(\widehat{\mathfrak{g}})$-module $\left(\rho_{c}, W_{\infty}\right)$ and $Y_{q}(\mathfrak{g})$-module $\left(\rho_{+}, W_{\infty}\right)$ respectively. For computational purpose the next observation is useful. ${ }^{7}$

Remark 5.3. Let us be in the situation of Section 2. For $x \in A$, one can find $K(x), L(x) \in \mathbb{Z}_{>0}$ (depending on $x$ and $L, K)$ such that: for all $l>0$ there exists a $\operatorname{Hom}\left(V_{l}, V_{l+L(x)}\right)$-valued Laurent polynomial $\sum_{i=-K(x)}^{K(x)} P_{x ; l}^{[i]} u^{i}=P_{x ; l}(u)$ in $u$ with

$$
\rho^{k}(x) F_{k, l}=\left.F_{k, l+L(x)} P_{x ; l}(u)\right|_{u=q^{k}} \in \operatorname{Hom}\left(V_{l}, V_{k}\right) \quad \text { for } \quad k>l+L(x) .
$$

In the representation of $A$ on $V_{\infty}, x$ acts as $\lim _{l \rightarrow \infty} P_{x ; l}(c)$. For example, let $x=s t$ with $s, t \in S$. Then $L(x)=2 L, K(x)=2 K$ and $P_{s t ; l}(u)=P_{s ; l+L}(u) P_{t ; l}(u)$.

To compute $x v$ with $v \in V_{\infty}$, one finds $l>0$ such that $v=F_{l}\left(v^{\prime}\right)$ for some $v^{\prime} \in V_{l}$; here $F_{l}: V_{l} \longrightarrow V_{\infty}$ is a structural map of the inductive limit. Then one writes $\rho^{k}(x) F_{k, l}\left(v^{\prime}\right)=$ $\left.F_{k, l+L(x)} \mathcal{P}_{l}(u)\right|_{u=q^{k}}$ for $k>l+L(x)$, where $\mathcal{P}_{l}(u)$ is a $V_{l+L(x)^{-v a l u e d ~ L a u r e n t ~ p o l y n o m i a l ~ i n ~} u \text { of }}$ degree bounded by $K(x)$. At last, $F_{l+L(x)}\left(\mathcal{P}_{l}(c)\right)$ is exactly $x v \in V_{\infty}$.

Consider the action of the $s_{i i}$ on $W_{a ; c}^{(r)}$. Let $v=F_{l}\left(v^{\prime}\right)$ with $v^{\prime} \in\left(W_{l, a}^{(r)}\right)_{l \varpi_{r}-\beta}$ and $\beta \in \mathbf{Q}_{+}$. We have $F_{k, l}\left(v^{\prime}\right) \in\left(W_{k, a}^{(r)}\right)_{k \varpi_{r}-\beta}$ and so $\rho^{k}\left(s_{i i}\right) F_{k, l}\left(v^{\prime}\right)=q^{\left(\epsilon_{i}, k \varpi_{r}-\beta\right)} F_{k, l}\left(v^{\prime}\right)$. This gives

$$
\rho_{c}\left(s_{i i}\right) v=c^{d_{r}\left(\epsilon_{i}, \varpi_{r}\right)} q^{\left(\epsilon_{i},-\beta\right)} v, \quad \rho_{+}\left(s_{i i}\right) v=q^{\left(\epsilon_{i},-\beta\right)} v .
$$

Let $\omega_{\infty}:=F_{1}\left(\omega_{1}\right) \in \omega_{\infty}$. Then $\rho_{+}\left(s_{i i}(z)\right) \omega_{\infty}=\omega_{\infty} \begin{cases}1-z a, & i \leq r \\ 1, & i>r .\end{cases}$

If $r \leq M$ then $\rho_{c}\left(s_{i i}(z)\right) \omega_{\infty}=\omega_{\infty}\left\{\begin{array}{ll}c-z a c, & i \leq r, \\ 1-z a c^{2}, & i>r .\end{array}\right.$ On the other hand for $r>M$ we have $\rho_{c}\left(s_{i i}(z)\right) \omega_{\infty}=\omega_{\infty}\left\{\begin{array}{ll}1-z a, & i \leq r, \\ c^{-1}-z a c, & i>r .\end{array}\right.$ In both $W_{a ; c}^{(r)}$ and $L_{r, a}^{+}, \omega_{\infty}$ is the unique (up to

\footnotetext{
${ }^{7}$ In [36], Drinfeld second realization arising from a different Gauss decomposition from Section 6 is used to resolve the issue in footnote 2 . This results in different parameterizations of highest $\ell$-weights. The two asymptotic limits of $\mathrm{KR}$ modules are denoted by $\mathcal{W}_{c, a}^{(r)}, L_{r, a}^{-}$therein and match with [21].
} 
scalar multiple) vector annihilated by the $\left(e_{i}^{+}\right)_{i \in I_{0}}$ and so by the $s_{i j}(z)$ for $i<j$, because of Proposition 4.3. A somewhat surprising observation from Table (5.1) is that $\rho_{+}\left(s_{i j}(z)\right)=0$ if $r<j<i$.

For the quantum affine superalgebra $U_{q}\left(\widehat{\mathfrak{g}^{\prime}}\right)$ in Proposition 3.2, one can define in the same way the KR modules $W_{k, a}^{\prime(r)}$ and construct their asymptotic limits $W_{a ; c}^{\prime(r)}, L_{r, a}^{\prime+}$.

Definition 5.4. The $Y_{q}(\mathfrak{g})$-module $L_{r, a}^{-}$is the pullback of the $Y_{q}\left(\mathfrak{g}^{\prime}\right)$-module $L_{M+N-r, a}^{\prime+}$ by the inverse $\mathcal{F}^{-1}$ of $\mathcal{F}: Y_{q}\left(\mathfrak{g}^{\prime}\right) \longrightarrow Y_{q}(\mathfrak{g})$ in Proposition 3.2.

As in the positive case, there is a unique (up to scalar multiple) vector $\omega_{\infty} \in L_{r, a}^{-}$annihilated by the $s_{i j}(z)$ for $i<j . \omega_{\infty}$ is of even parity and $\rho_{-}\left(s_{i i}(z)\right) \omega_{\infty}=\omega_{\infty}\left\{\begin{array}{ll}1, & i \leq r, \\ 1-z a, & i>r .\end{array}\right.$ We shall see that $L_{r, a}^{ \pm}$are irreducible $Y_{q}(\mathfrak{g})$-modules.

Example $5.5(\mathfrak{g}=\mathfrak{g l}(2,1)$ and $r=1)$. Let $k>0$. Fix a highest $\ell$-weight vector $u_{0}$ of $W_{k, a}^{(1)}$. By Theorem 3.4, for $1 \leq i \leq k$, there exist unique $u_{i}, u_{i}^{\prime}$ such that $u_{0}=\left(e_{1}^{+}\right)^{i} u_{i}$ and $u_{i}=e_{2}^{+} u_{i}^{\prime}$. These together with $u_{0}$ form a basis of $W_{k, a}^{(1)}$ of weight

$$
u_{i} \in\left(W_{k, a}^{(1)}\right)_{(k-i) \epsilon_{1}+i \epsilon_{2}}, \quad u_{i}^{\prime} \in\left(W_{k, a}^{(1)}\right)_{(k-i) \epsilon_{1}+(i-1) \epsilon_{2}+\epsilon_{3}} .
$$

The structural maps $F_{k, l}$ respect these bases because they commute with $e_{1}^{+}, e_{2}^{+}$.

Firstly compute the action of $\left(e_{i}^{ \pm}, K_{i}\right)$. By weight grading, $e_{1}^{-} u_{i}=a_{i} u_{i+1}$ with $a_{i} \in \mathbb{C}$ for $0 \leq i<k$. From $u_{i}=e_{1}^{+} u_{i+1}$ and relation (3.3) we obtain

$$
a_{i} u_{i+1}=e_{1}^{-} e_{1}^{+} u_{i+1}=e_{1}^{+} e_{1}^{-} u_{i+1}-\frac{K_{1}-K_{1}^{-1}}{q-q^{-1}} u_{i+1}=\left(a_{i+1}-\frac{q^{k-2 i-2}-q^{2 i+2-k}}{q-q^{-1}}\right) u_{i+1} .
$$

By convention $a_{k}:=0$. This recurrence gives $a_{i}=\frac{\left(q^{k}-q^{2 i-k}\right)\left(q-q^{-2 i-1}\right)}{\left(q-q^{-1}\right)^{2}}$.

From $e_{2}^{+} e_{1}^{-} u_{i}^{\prime}=e_{1}^{-} e_{2}^{+} u_{i}^{\prime}=e_{1}^{-} u_{i}=a_{i} u_{i+1}$ follows also $e_{1}^{-} u_{i}^{\prime}=a_{i} u_{i+1}^{\prime}$. Noting $e_{2}^{+} u_{i}^{\prime}=u_{i}$ and $e_{2}^{-} u_{i}^{\prime}=0$ (because of the weight grading), $e_{2}^{-} u_{i}=e_{2}^{-} e_{2}^{+} u_{i}^{\prime}=\frac{K_{2}-K_{2}^{-1}}{q-q^{-1}} u_{i}^{\prime}=\frac{q^{i}-q^{-i}}{q-q^{-1}} u_{i}^{\prime}$. Applying $e_{1}^{+}$ to this identity and using $\left[e_{1}^{+}, e_{2}^{-}\right]=0$, we have $e_{1}^{+} u_{i}^{\prime}=\frac{q^{i-1}-q^{1-i}}{q^{i}-q^{-i}} u_{i-1}^{\prime}$.

Secondly consider $s_{13}$ and $t_{31}$. In view of relation (3.4) we have

$$
\begin{aligned}
& s_{13}=\frac{s_{11}}{q-q^{-1}}\left(q e_{1}^{+} e_{2}^{+}-e_{2}^{+} e_{1}^{+}\right), \quad t_{31}=\left(e_{1}^{-} e_{2}^{-}-q^{-1} e_{2}^{-} e_{1}^{-}\right) \frac{t_{11}}{q-q^{-1}}, \\
& s_{13} u_{i}^{\prime}=\frac{s_{11}}{q-q^{-1}}\left(q-\frac{q^{i-1}-q^{1-i}}{q^{i}-q^{-i}}\right) u_{i-1}=\frac{q^{k+1}}{q^{i}-q^{-i}} u_{i-1}, \\
& t_{31} u_{i}=\frac{q^{i-k}}{q-q^{-1}}\left(a_{i} \frac{q^{i}-q^{-i}}{q-q^{-1}}-q^{-1} \frac{q^{i+1}-q^{-i-1}}{q-q^{-1}} a_{i}\right) u_{i+1}^{\prime}=-\frac{\left(1-q^{2 i-2 k}\right)\left(1-q^{-2 i-2}\right)}{\left(q-q^{-1}\right)^{3}} u_{i+1}^{\prime} .
\end{aligned}
$$

Let $E_{i j} \in$ End $\left(W_{k, a}^{(1)}\right)$ with $i, j \in\{0,1, \ldots, k, \overline{1}, \ldots, \bar{k}\}$ be elementary matrices with respect to the basis $\left(u_{i}, u_{i}^{\prime}\right)$. In summary, the $U_{q}(\widehat{\mathfrak{g}})$-module structure on $W_{k, a}^{(1)}$ is given by

$$
\begin{aligned}
& \rho^{k}\left(s_{11}(z)\right)=q^{k} \times\left(\sum_{i=0}^{k}\left(q^{-i}-z a q^{i}\right) E_{i i}+\sum_{i=1}^{k}\left(q^{-i}-z a q^{i}\right) E_{\bar{i} i}\right), \\
& \rho^{k}\left(s_{22}(z)\right)=\sum_{i=0}^{k}\left(q^{i}-z a q^{2 k-i}\right) E_{i i}+\sum_{i=1}^{k}\left(q^{i-1}-z a q^{2 k-i+1}\right) E_{\bar{i} i},
\end{aligned}
$$




$$
\begin{aligned}
& \rho^{k}\left(s_{33}(z)\right)=\sum_{i=0}^{k}\left(1-z a q^{2 k}\right) E_{i i}+\sum_{i=1}^{k}\left(q^{-1}-z a q^{2 k+1}\right) E_{\overline{i i}}, \\
& \rho^{k}\left(s_{12}(z)\right)=q^{k} \times\left(\sum_{i=0}^{k-1} q^{-i} E_{i, i+1}+\sum_{i=1}^{k-1} \frac{1-q^{-2 i}}{q^{i+1}-q^{-i-1}} E_{\bar{i}, \overline{i+1}}\right), \\
& \rho^{k}\left(s_{13}(z)\right)=q^{k} \times \sum_{i=1}^{k} \frac{q}{q^{i}-q^{-i}} E_{i-1, \bar{i}}, \\
& \rho^{k}\left(s_{23}(z)\right)=\sum_{i=1}^{k} q^{i} E_{i, \bar{i}}, \\
& \rho^{k}\left(s_{21}(z)\right)=z a \sum_{i} \frac{\left(q^{2 i}-q^{2 k}\right)\left(q^{i+1}-q^{-i-1}\right)}{\left(q-q^{-1}\right)^{2}}\left(E_{i+1, i}+E_{\overline{i+1}, \bar{i}}\right), \\
& \rho^{k}\left(s_{31}(z)\right)=z a \sum_{i=0}^{k-1} \frac{\left(q^{2 k}-q^{2 i}\right)\left(1-q^{-2 i-2}\right)}{\left(q-q^{-1}\right)^{3}} E_{\overline{i+1}, i}, \\
& \rho^{k}\left(s_{32}(z)\right)=z a \sum_{i=1}^{k} \frac{q^{2 k-2 i}-q^{2 k}}{q-q^{-1}} E_{\bar{i}, i} .
\end{aligned}
$$

Here for $\rho^{k}\left(s_{21}(z)\right)$, the summation $\sum_{i}$ is understood to be $0 \leq i<k$ for $E_{i+1, i}$ and $1 \leq i<k$ for $E_{\overline{i+1}, \bar{i}}$. Letting $k \rightarrow \infty$ and replacing $\left(\rho^{k}, q^{k}\right)$ in the above formulas by $\left(\rho_{c}, c\right)$ gives the $U_{q}(\widehat{\mathfrak{g}})$ module $W_{a ; c}^{(1)}$. (Note that $\rho_{c}\left(t_{i j}^{(n)}\right)=-a^{-1} c^{-2} \rho_{c}\left(s_{i j}^{(1-n)}\right)$.) Dividing $\rho^{k}\left(s_{i j}(z)\right)$ by $q^{k}$ whenever $i \leq 1$ and then setting $q^{k}=0$, we obtain the $Y_{q}(\mathfrak{g})$-module $L_{1, a}^{+}$.

In terms of Young diagrams, the $u_{i}, u_{i}^{\prime}$ correspond to the following tableaux (let $k=3$ )

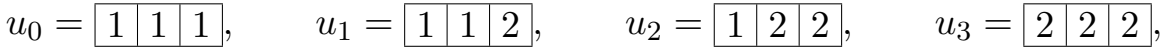

$$
\begin{aligned}
& u_{1}^{\prime}=\begin{array}{l|l|l}
1 & 1 & 3
\end{array}, \quad u_{2}^{\prime}=\begin{array}{l|l|l|l}
1 & 2 & 3
\end{array}, \quad u_{3}^{\prime}=\begin{array}{ll|l|l|}
2 & 2 & 3 \\
\hline
\end{array} .
\end{aligned}
$$

Example 5.6 $(\mathfrak{g}=\mathfrak{g l}(2,1)$ and $r=2)$. For $k>0$, let $u_{4} \in W_{k, a}^{(2)}$ be such that $e_{i}^{-} u_{4}=0$ for $i=1,2$. It is included in a basis $\left(u_{1}, u_{2}, u_{3}, u_{4}\right)$ of $W_{k, a}^{(2)}$ by Theorem 3.4:

$$
u_{3}:=e_{2}^{+} u_{4}, \quad u_{2}:=e_{1}^{+} u_{3}, \quad u_{1}:=e_{2}^{+} u_{2} .
$$

From relations (3.3)-(3.5), one deduces the action of the $e_{i}^{ \pm}, K_{i}$ and then $U_{q}(\mathfrak{g})$. We identify the vector superspace $W_{k, a}^{(2)}$ with $W_{1, a}^{(2)}$ by this basis. The structure maps $F_{k, l}$ are identity maps because they commute with the $e_{i}^{+}$. Let $E_{i j} \in \operatorname{End}\left(W_{k, a}^{(2)}\right): u_{k} \mapsto u_{i} \delta_{j k}$. Then

$$
\begin{aligned}
& \rho^{k}\left(s_{11}(z)\right)=q^{k} \times\left((1-z a)\left(E_{11}+E_{22}\right)+\left(q^{-1}-z a q\right)\left(E_{33}+E_{44}\right)\right), \\
& \rho^{k}\left(s_{22}(z)\right)=q^{k} \times\left((1-z a)\left(E_{11}+E_{33}\right)+\left(q^{-1}-z a q\right)\left(E_{22}+E_{44}\right)\right), \\
& \rho^{k}\left(s_{33}(z)\right)=\left(1-z a q^{2 k}\right) E_{11}+\left(q^{-1}-z a q^{2 k+1}\right)\left(E_{22}+E_{33}\right)+\left(q^{-2}-z a q^{2 k+2}\right) E_{44}, \\
& \rho^{k}\left(s_{12}(z)\right)=q^{k} \times\left(1-q^{-2}\right) E_{23}, \\
& \rho^{k}\left(s_{13}(z)\right)=q^{k} \times\left(q^{-1}-q^{-3}\right)\left(q E_{24}-E_{13}\right), \\
& \rho^{k}\left(s_{23}(z)\right)=q^{k} \times\left(1-q^{-2}\right)\left(E_{12}+E_{34}\right), \\
& \rho^{k}\left(s_{21}(z)\right)=q^{k} \times z a\left(q^{2}-1\right) E_{32}, \\
& \rho^{k}\left(s_{31}(z)\right)=z a\left(q^{2 k+2}-1\right) E_{42}-z a\left(q^{2 k+2}-q^{2}\right) E_{31},
\end{aligned}
$$




$$
\rho^{k}\left(s_{32}(z)\right)=z a\left(q^{2 k+1}-q\right) E_{21}+z a\left(q^{2 k+2}-1\right) E_{43} .
$$

The modules $W_{c ; a}^{(2)}, L_{2, a}^{+}$are then obtained as in the previous example.

Again in terms of tableaux $u_{1}, u_{2}, u_{3}, u_{4}$ correspond to (let $k=3$ )

$$
u_{1}=\begin{array}{|l|l|l|}
\hline 1 & 1 & 1 \\
\hline 2 & 2 & 2
\end{array}, \quad u_{2}=\begin{array}{|l|l|l|}
\hline 1 & 1 & 1 \\
\hline 2 & 2 & 3 \\
\hline
\end{array}, \quad u_{3}=\begin{array}{|l|l|l|}
1 & 1 & 2 \\
\hline 2 & 2 & 3 \\
\hline
\end{array}, \quad u_{4}=\begin{array}{|l|l|l|}
\hline 1 & 1 & 3 \\
\hline 2 & 2 & 3 \\
\hline
\end{array} .
$$

Remark 5.7. The $L_{r, a}^{ \pm}$were previously obtained in [7,31] from the contracted quantum superalgebra $\dot{U}_{q}(\mathfrak{g})$. It is a superalgebra defined in the same as $U_{q}(\mathfrak{g})$ in Definition 3.1 except that the $t_{i i}$ are not required to be invertible. $\mathrm{ev}_{a}$ in Proposition 3.2 degenerates to $\operatorname{ev}_{a}: Y_{q}(\mathfrak{g}) \longrightarrow \dot{U}_{q}(\mathfrak{g})$. The $L_{r, a}^{ \pm}$are pullbacks of oscillator modules over $\dot{U}_{q}(\mathfrak{g})$ by $\dot{\mathrm{ev}}_{a}$.

Lemma 5.8. Let $\beta \in \mathbf{Q}_{+}$. The series $\operatorname{dim}\left(W_{k, a}^{(r)}\right)_{k \varpi_{r}-\beta}$ converges as $k \rightarrow \infty$.

Proof. Let $U_{q}^{-}(\mathfrak{g})$ be the subalgebra of $U_{q}(\mathfrak{g})$ generated by the $\left(e_{i}^{-}\right)_{i \in I_{0}}$. Then $U_{q}(\mathfrak{g})_{-\beta}$ is of dimension $c_{\beta}<\infty$. By definition, $U_{q}^{-}(\mathfrak{g}) \longrightarrow W_{k, a}^{(r)}, x \mapsto x \omega_{k}$ is surjective and sends $U_{q}(\mathfrak{g})_{-\beta}$ to $\left(W_{k, a}^{(r)}\right)_{k \varpi_{r}-\beta}$. This shows that the series is bounded above by $c_{\beta}$. On the other hand it is increasing by Proposition 4.3. So it must converge.

\section{Category $\mathcal{O}$ and Baxter's relations}

We introduce a category $\mathcal{O}$ of $Y_{q}(\mathfrak{g})$-modules including $W_{a ; b}^{(r)}$ and $L_{r, a}^{ \pm}$in the spirit of HernandezJimbo [21] and study its Grothendieck ring via $q$-characters of [16].

The quantum affine superalgebra $U_{q}(\widehat{\mathfrak{g}})$ admits another system of generators, the so-called Drinfeld loop generators, arising from the Gauss decomposition:

$$
\begin{aligned}
& S(z)=\left(\sum_{i<j} f_{j i}^{+}(z) \otimes E_{j i}+1 \otimes \mathrm{Id}_{\mathbf{V}}\right)\left(\sum_{l} K_{l}^{+}(z) \otimes E_{l l}\right)\left(\sum_{i<j} e_{i j}^{+}(z) \otimes E_{i j}+1 \otimes \mathrm{Id}_{\mathbf{V}}\right), \\
& T(z)=\left(\sum_{i<j} f_{j i}^{-}(z) \otimes E_{j i}+1 \otimes \mathrm{Id}_{\mathbf{V}}\right)\left(\sum_{l} K_{l}^{-}(z) \otimes E_{l l}\right)\left(\sum_{i<j} e_{i j}^{-}(z) \otimes E_{i j}+1 \otimes \mathrm{Id}_{\mathbf{V}}\right) .
\end{aligned}
$$

For example, $K_{1}^{+}(z)=s_{11}(z)$ and $K_{1}^{-}(z)=t_{11}(z)$. We refer to [34, Section 3] for more details on the relations and on coproduct formulas of these Drinfeld generators. Recall the $d_{i}$ from equation (3.1). Define $\theta_{i}$ for $i \in I$ inductively by $\theta_{1}=1, \theta_{i+1}=q_{i+1} q_{i} \theta_{i}$. Define

$$
C_{i}(z):=\prod_{j=1}^{i} K_{j}^{+}\left(z \theta_{j}\right)^{d_{j}}=\sum_{n \geq 0} C_{i, n} z^{n}, \quad K_{i}^{+}(z)=: \sum_{n \geq 0} K_{i, n}^{+} z^{n} \in Y_{q}(\mathfrak{g})[[z]] .
$$

Proposition 6.1 ([34, Theorem 3.5, Proposition 3.6]). Let $k \in I$ and $m, n \geq 0$.

(1) $\Delta\left(K_{k, m}^{+}\right)-\sum_{l=0}^{m} K_{k, l}^{+} \otimes K_{k, m-l}^{+} \in \sum_{\alpha \in \mathbf{Q}_{+} \backslash\{0\}} Y_{q}(\mathfrak{g})_{\alpha} \otimes Y_{q}(\mathfrak{g})_{-\alpha}$.

(2) For all $i, j \in I$ such that $i, j \leq k$, the $s_{i j}^{(n)}, t_{i j}^{(n)}$ commute with $C_{i, m}$.

Set $\mathfrak{P}:=\left(\mathbb{C}^{\times}\right)^{I} \times \mathbb{Z}_{2}$, and $\widehat{\mathfrak{P}}:=\left(\mathbb{C}[[z]]^{\times}\right)^{I} \times \mathbb{Z}_{2}$. The multiplicative group structure on $\mathbb{C}^{\times}, \mathbb{C}[[z]]^{\times}$and the additive group structure on the ring $\mathbb{Z}_{2}$ make $\mathfrak{P}, \widehat{\mathfrak{P}}$ into multiplicative abelian groups. $\mathfrak{P}$ is naturally a subgroup of $\widehat{\mathfrak{P}}$, and $\mathbb{C}[[z]]^{\times} \longrightarrow \mathbb{C}^{\times}, h(z) \mapsto h(0)$ induces a projection $\varpi: \widehat{\mathfrak{P}} \longrightarrow \mathfrak{P}$. There is an injective homomorphism of abelian groups

$$
q^{?}: \mathbf{P} \longrightarrow \mathfrak{P}, \quad \lambda \mapsto q^{\lambda}:=\left(\left(q^{\left(\epsilon_{i}, \lambda\right)}\right)_{i \in I} ;|\lambda|\right) .
$$

We shall view $h(z) \in \mathbb{C}[[z]]^{\times}$as the element $\left(\left(h_{i}(z)=h(z)\right)_{i \in I} ; \overline{0}\right)$ in $\widehat{\mathfrak{P}}$. 
Let $V$ be a $U_{q}(\mathfrak{g})$-module. For $p=\left(\left(p_{i}\right)_{i \in I} ; s\right) \in \mathfrak{P}$, define

$$
V_{p}:=\left\{v \in V_{s} \mid s_{i i} v=p_{i} v \text { for } i \in I\right\} .
$$

If $V_{p} \neq 0$, then $p$ is called a weight of $V$, and $V_{p}$ the weight space of weight $p$. Let wt $(V)$ denote the set of weights of $V$. Notice that $U_{q}(\mathfrak{g})_{\alpha} V_{p} \subseteq V_{q^{\alpha}}$ for $p \in \operatorname{wt}(V)$ and $\alpha \in \mathbf{Q}$.

Lemma 6.2. For $\lambda, \mu \in \mathbf{P}$, the weight space $L(\lambda)_{\mu}$ in (3.6) and $L(\lambda)_{q^{\mu}}$ in (6.1) coincide.

Proof. Clearly $L(\lambda)_{q^{\mu}} \subseteq L(\lambda)_{\mu}$ for all $\mu \in \mathbf{P}$. Furthermore $L^{\prime}:=\oplus_{\mu \in \mathbf{P}} L(\lambda)_{q^{\mu}}$ is easily seen to be a sub- $U_{q}(\mathfrak{g})$-module. Since $v_{\lambda} \in L^{\prime}$ and since $L(\lambda)$ is irreducible, $L(\lambda)=L^{\prime}$. This implies $L(\lambda)_{q^{\mu}}=L(\lambda)_{\mu}$ for all $\mu \in \mathbf{P}$.

Let $V$ be a $Y_{q}(\mathfrak{g})$-module. For $\mathbf{f}=\left(\left(f_{i}(z)\right)_{i \in I} ; s\right) \in \widehat{\mathfrak{P}}$ define

$$
V_{\mathbf{f}}:=\left\{v \in V_{s} \mid \exists d \in \mathbb{Z}_{>0} \text { such that }\left(K_{i}^{+}(z)-f_{i}(z)\right)^{d} v=0 \text { for } i \in I\right\} .
$$

If $V_{\mathbf{f}} \neq 0$, then $\mathbf{f}$ is called an $\ell$-weight of $V$, and $V_{\mathbf{f}}$ the $\ell$-weight space of $\ell$-weight $\mathbf{f}$. Let wt $t_{\ell}(V)$ be the set of $\ell$-weights of $V$. As in Section 4 , a non-zero $\mathbb{Z}_{2}$-homogeneous vector $v$ in a $Y_{q}(\mathfrak{g})$ module $V$ is called a highest $\ell$-weight vector if $s_{j k}^{(n)} v=0$ for $j<k$ and $s_{i i}(z) v=g_{i}(z) v$ with $g_{i}(z) \in \mathbb{C}[[z]]^{\times}$. By Gauss decomposition we have $K_{i}^{+}(z) v=g_{i}(z) v$, so $v$ is in the $\ell$-weight space of $\ell$-weight $\left(\left(g_{i}(z)\right)_{i \in I} ;|v|\right)$. If furthermore $V=Y_{q}(\mathfrak{g}) v$, then $V$ is called a highest $\ell$-weight module, and $\left(\left(g_{i}(z)\right)_{i \in I} ;|v|\right)$ the highest $\ell$-weight of $V$.

By the comments above Definition 5.4, any one the $Y_{q}(\mathfrak{g})$-modules $W_{k, a}^{(r)}, W_{a ; c}^{(r)}, L_{r, a}^{ \pm}$contains a unique highest $\ell$-weight vector $\omega_{\infty}$, and its $\ell$-weight is denoted by $\mathbf{w}_{k, a}^{(r)}, \mathbf{w}_{a ; c}^{(r)}, \mathbf{w}_{r, a}^{ \pm}$correspondingly. Explicitly, $\mathbf{w}_{k, a}^{(r)}=\mathbf{w}_{a ; q_{r}^{k}}^{(r)}, \mathbf{w}_{a ; 1}^{(r)}=1-z a$ and

$$
\begin{aligned}
& \mathbf{w}_{r, a}^{+}=(\underbrace{1-z a, \ldots, 1-z a}_{r}, \underbrace{1, \ldots, 1}_{M+N-r} ; \overline{0}), \quad \mathbf{w}_{r, a}^{-}=(\underbrace{1, \ldots, 1}_{r}, \underbrace{1-z a, \ldots, 1-z a}_{M+N-r} ; \overline{0}), \\
& \begin{array}{|c|c|c|}
\hline & 1, \ldots>M \\
\hline \frac{\mathbf{w}_{a c}^{(r)}}{\mathbf{w}_{a ; 1}^{(r)}} & (\underbrace{c, \ldots, c}_{r}, \underbrace{g(z), \ldots, g(z)}_{M+N-r} ; \overline{0}) & (\underbrace{1, \ldots, 1}_{r}, \underbrace{h(z), \ldots, h(z)}_{M+N-r} ; \overline{0}) \\
\hline & g(z)=\frac{1-z a c^{2}}{1-z a} & h(z)=\frac{c^{-1}-z a c}{1-z a} \\
\hline
\end{array}
\end{aligned}
$$

Definition 6.3. Category $\mathcal{O}$ is a full subcategory of the category of $Y_{q}(\mathfrak{g})$-modules. An object of $\mathcal{O}$ is a $Y_{q}(\mathfrak{g})$-module $V$ subject to the following conditions:

(i) it has a weight space decomposition $V=\oplus_{p \in \mathfrak{P}} V_{p}$ with $\operatorname{dim} V_{p}<\infty$ for all $p \in \mathfrak{P}$;

(ii) there exist $\mu_{1}, \mu_{2}, \ldots, \mu_{d} \in \mathfrak{P}$ such that $\operatorname{wt}(V) \subseteq \cup_{j=1}^{d}\left(q^{\mathbf{Q}_{-}} \mu_{j}\right)$.

As a first example, let $p=\left(\left(p_{i}\right)_{i \in I} ; s\right) \in \mathfrak{P}$ and $h(z) \in \mathbb{C}[[z]]^{\times}$. There exists a unique $Y_{q}(\mathfrak{g})$ module structure, denoted by $\mathbb{C}^{h(z) p}$, on the one-dimensional vector superspace of parity $s$ such that $s_{i j}^{(n)}=\delta_{n 0} \delta_{i j} h(z) p_{i}$. Clearly wt $\operatorname{w}_{\ell}\left(\mathbb{C}^{h(z) p}\right)=\{h(z) p\}$.

By Corollary 5.2 and Lemma 5.8, the $Y_{q}(\mathfrak{g})$-modules $W_{a ; c}^{(r)}, L_{r, a}^{ \pm}$are in category $\mathcal{O}$.

Category $\mathcal{O}$ is monoidal (closed under tensor products) and abelian. Any $Y_{q}(\mathfrak{g})$-module $V$ in category $\mathcal{O}$ is a direct sum of its $\ell$-weight spaces and has $q$-character [16]

$$
\chi_{q}(V)=\sum_{\mathbf{f} \in \mathrm{wt}_{\ell}(V)} \operatorname{dim}\left(V_{\mathbf{f}}\right) \mathbf{f} \in \mathcal{E}_{\ell} .
$$


Here the target $\mathcal{E}_{\ell}$ is the set of formal sums $\sum_{\mathbf{f} \in \widehat{\mathfrak{P}}} n_{\mathbf{f}} \mathbf{f}$ of the symbols $\mathbf{f}$ with integer coefficients $n_{\mathbf{f}}$ such that $\oplus_{\mathbf{f} \in \widehat{\mathfrak{P}}}\left(\mathbb{C}^{\varpi(\mathbf{f})}\right) \oplus\left|n_{\mathbf{f}}\right|$ is in category $\mathcal{O}$. It admits a ring structure: addition is the usual one of formal sums; multiplication is induced by that of $\widehat{\mathfrak{P}}$.

Replacing $\mathrm{wt}_{\ell}$ by wt in equation (6.4) defines the classical character $\chi(V)$.

Lemma 6.4. We have $\chi_{q}(V \otimes W)=\chi_{q}(V) \chi_{q}(W)$ for $V, W$ in category $\mathcal{O}$.

Proof. The proof is the same as in the non-graded case [16, Remark 2.6] based on Proposition 6.1(1) and the partial order on $\mathfrak{P}$ induced by $q^{\mathbf{Q}_{+}}$.

Remark 6.5. Let $V$ be in category $\mathcal{O}$. Suppose there exists $p \in \mathfrak{P}$ such that the weight space $V_{p}$ is one-dimensional and $\operatorname{wt}(V) \subseteq q^{\mathbf{Q}_{-}} p$. Then $V_{p}$ is also an $\ell$-weight space of $\ell$-weight $\mathbf{f}$. Define the normalized character and normalized $q$-character of $V$ by

$$
\tilde{\chi}(V):=p^{-1} \chi(V), \quad \tilde{\chi}_{q}(V):=\mathbf{f}^{-1} \chi_{q}(V) .
$$

This is the case when $V$ is any tensor product of the $W_{a ; c}^{(r)}, L_{r, a}^{ \pm}$. If $D$ is a one-dimensional $Y_{q}(\mathfrak{g})$-module, then the normalized $q$-characters of $V, V \otimes D, D \otimes V$ coincide.

Remark 6.6. Let $V, W$ be in category $\mathcal{O}$. Let $v \in V$ be a highest $\ell$-weight vector of $\ell$-weight $\mathbf{f} \in \widehat{\mathfrak{P}}$. Then $v \otimes W_{\mathbf{n}} \subseteq(V \otimes W)_{\mathbf{n f}}$ for $\mathbf{n} \in \mathrm{wt}_{\ell}(W)$. This follows from Proposition $6.1(1)$; the term $Y_{q}(\mathfrak{g})_{\alpha} v$ for $\alpha \in \mathbf{Q}_{+} \backslash\{0\}$ vanishes.

Lemma 6.7. Let $r \in I_{0}$ and $a, c \in \mathbb{C}^{\times}$. Then $\widetilde{\chi}_{q}\left(W_{k, a}^{(r)}\right)$ converges in $\mathcal{E}_{\ell}$ as $k \rightarrow \infty$, and

$$
\begin{aligned}
& \tilde{\chi}\left(W_{a ; c}^{(r)}\right)=\lim _{k \rightarrow \infty} \tilde{\chi}\left(W_{k, a}^{(r)}\right)=\tilde{\chi}\left(L_{r, a}^{+}\right)=\chi\left(L_{r, a}^{+}\right), \\
& \tilde{\chi}_{q}\left(W_{a ; c}^{(r)}\right)=\lim _{k \rightarrow \infty} \tilde{\chi}_{q}\left(W_{k, a}^{(r)}\right)=\tilde{\chi}_{q}\left(L_{r, a}^{+}\right) .
\end{aligned}
$$

Proof. We simplify notation $\mathbf{w}_{k, a}^{(r)}=: \mathbf{w}^{k} \in \widehat{\mathfrak{P}}$. Let $\mathbf{n} \in \widehat{\mathfrak{P}}$ with $\mathbf{w}^{l} \mathbf{n} \in \mathrm{wt}_{\ell}\left(W_{l, a}^{(r)}\right)$. Then $\varpi(\mathbf{n})=q^{-\beta}$ with $\beta \in \mathbf{Q}_{+}$. By Remark 6.6, $F_{k, l}\left(W_{l, a}^{(r)}\right)_{\mathbf{w}^{l} \mathbf{n}} \subseteq\left(W_{k, a}^{(r)}\right)_{\mathbf{w}^{k} \mathbf{n}}$. So the series $\left\{\operatorname{dim}\left(W_{k, a}^{(r)}\right)_{\mathbf{w}^{k} \mathbf{n}}: k>0\right\}$ is increasing and bounded by $c_{\beta}$ in the proof of Lemma 5.8. This proves the convergence of the $\tilde{\chi}_{q}\left(W_{k, a}^{(r)}\right)$ as $k \rightarrow \infty$.

Next, fix $\mathbf{n}=\left(\left(n_{i}(z)\right)_{i \in I} ;|\beta|\right)$ with $\varpi(\mathbf{n})=q^{-\beta}$ and $0 \neq x \in\left(W_{l, a}^{(r)}\right)_{\mathbf{w}^{l} \mathbf{n}}$. For $k>l$ write $\mathbf{w}^{k}=\left(\left(w_{i}^{k}(z)\right)_{i \in I} ; \overline{0}\right) \in \widehat{\mathfrak{P}}$. Since the $\ell$-weight space $\left(W_{k, a}^{(r)}\right)_{\mathbf{w}^{k} \mathbf{n}}$ is of dimension $\leq c_{\beta}$,

$$
\rho^{k}\left(K_{i}^{+}(z)-h(z)\right)^{c_{\beta}} F_{k, l}(x)=\left(w_{i}^{k}(z) n_{i}(z)-h(z)\right)^{c_{\beta}} F_{k, l}(x) \quad \text { for } \quad h(z) \in \mathbb{C}[[z]] .
$$

Indeed, because of the commutativity of its coefficients, $\rho^{k}\left(K_{i}^{+}(z)\right)$ restricted to $\left(W_{k, a}^{(r)}\right)_{\mathbf{w}^{k} \mathbf{n}}$ can be made into an upper triangular matrix with uniform diagonals $w_{i}^{k}(z) n_{i}(z)$.

Let us take $h(z)$ to be $n_{i}(z)$ times the $i$-th component $w_{i}(z)$ of $\mathbf{w}_{a ; c}^{(r)}$ :

$$
\rho^{k}\left(K_{i}^{+}(z)-w_{i}(z) n_{i}(z)\right)^{c_{\beta}} F_{k, l}(x)=\left(w_{i}^{k}(z)-w_{i}(z)\right)^{c_{\beta}} n_{i}(z)^{c_{\beta}} F_{k, l}(x) .
$$

At the right-hand side the factor before $F_{k, l}(x)$, when expanded at $z=0$, has as coefficients Laurent polynomials in $q_{r}^{k}$. To compute $\rho_{c}\left(K_{i}^{+}(z)-w_{i}(z) n_{i}(z)\right)^{c_{\beta}} F_{l}(x)$ in $W_{a ; c}^{(r)}$, it suffices to evaluate these polynomials at $q_{r}^{k}=c$ by Remark 5.3. But by definition the factor $\left(w_{i}^{k}(z)-\right.$ $\left.w_{i}(z)\right)\left.\right|_{q_{r}^{k}=c}=0$. So $\rho_{c}\left(K_{i}^{+}(z)-w_{i}(z) n_{i}(z)\right)^{c_{\beta}} F_{l}(x)=0$, meaning that $F_{l}(x) \in W_{a ; c}^{(r)}$ is in the $\ell$-weight space of $\ell$-weight $\mathbf{w}_{a ; c}^{(r)} \mathbf{n}$. This proves the first equality of equation (6.6). The second equality for $L_{r, a}^{+}$can be proved in the same way using $\tilde{\rho}^{k}$. 
Let $\mathbf{R}$ be the subset of $\widehat{\mathfrak{P}}$ consisting of the $\mathbf{f}=\left(\left(f_{i}(z)\right)_{i \in I} ; s\right)$ such that $\frac{f_{i}(z)}{f_{i+1}(z)}$ is the Taylor expansion at $z=0$ of a rational function for $i \in I_{0}$.

Lemma 6.8. Let $\mathbf{f}=\left(\left(f_{i}(z)\right)_{i \in I} ; s\right) \in \mathbf{R}$.

(1) There exists a unique (up to isomorphism) irreducible $Y_{q}(\mathfrak{g})$-module in category $\mathcal{O}$ of highest $\ell$-weight $\mathbf{f}$. Let $V(\mathbf{f})$ be the $Y_{q}(\mathfrak{g})$-module thus obtained.

(2) $V(\mathbf{f})$ can be extended to a $U_{q}(\widehat{\mathfrak{g}})$-module if and only if for all $i \in I_{0}$, as a rational function $\frac{f_{i}(z)}{f_{i+1}(z)}$ is a product of the $\frac{1-z a}{c^{-1}-z a c}$ with $a, c \in \mathbb{C}^{\times}$.

(3) All irreducible $Y_{q}(\mathfrak{g})$-modules in category $\mathcal{O}$ are of the form $V(\mathbf{f})$ with $\mathbf{f} \in \mathbf{R}$.

Proof. (1) and sufficiency of (2). In view of equations (6.2)-(6.3), such an $\mathbf{f}$ can be written as $h(z) p \mathbf{n}$ where $h(z) \in \mathbb{C}[[z]]^{\times}, p \in \mathfrak{P}$ (resp. $p$ is of the form $(1, \ldots, 1 ; s) \in \mathfrak{P}$ ) and $\mathbf{n}$ is a product of the $\mathbf{w}_{r, a}^{ \pm}$(resp. the $\mathbf{w}_{a ; c}^{(r)}$ ). So $V(\mathbf{f})$ can be realized as a sub-quotient of the tensor product of $V(h(z) p)$, which is a one-dimensional $Y_{q}(\mathfrak{g})$-module (resp. $U_{q}(\widehat{\mathfrak{g}})$-module), with the corresponding tensor product of the $L_{r, a}^{ \pm}$(resp. the $\left.W_{a ; c}^{(r)}\right)$. This shows that $V(\mathbf{f})$ is in category $\mathcal{O}$ (resp. a $U_{q}(\widehat{\mathfrak{g}})$-module).

Necessity of (2). One considers the action of $K_{i+1}^{ \pm}(z) K_{i}^{ \pm}(z)^{-1}$, based on the Drinfeld relations involving $\left[X_{i}^{+}, X_{i}^{-}\right]$in [34, Theorem 3.5] and the assumption $\operatorname{dim} V(\mathbf{f})_{\varpi(\mathbf{f}) q^{-\alpha_{i}}}<\infty$. As in [33, Proposition 6.1, Lemma 4.12], $g_{i}(z):=\frac{f_{i}(z)}{f_{i+1}(z)}$ is regular at $z=0, \infty$ and $g_{i}(0) g_{i}(\infty)=1$. Necessarily $g_{i}(z)$ is a product of the $\frac{1-z a}{c^{-1}-z a c}$ with $a, c \in \mathbb{C}^{\times}$.

Similar arguments can be used to prove (3); since $K_{i}^{-}(z) \notin Y_{q}(\mathfrak{g})\left[\left[z^{-1}\right]\right]$, one loses the regularity of $g_{i}(z)$ at $z=\infty$. See also [21, Lemma 3.9].

The abelian category $\mathcal{O}$ contains modules with infinite Jordan-Hölder series, so we need its completed Grothendieck group $K_{0}(\mathcal{O})$ : elements are formal sums $\sum_{\mathbf{f} \in \mathbf{R}} n_{\mathbf{f}}[V(\mathbf{f})]$ of the symbols $[V(\mathbf{f})]$ with integer coefficients $n_{\mathbf{f}}$ such that $\oplus_{\mathbf{f} \in \mathbf{R}} V(\mathbf{f})^{\oplus\left|n_{\mathbf{f}}\right|}$ is in category $\mathcal{O}$; addition is the usual one of formal sums. As in the case of Kac-Moody algebras [23, Section 9.3], for $\mathbf{f} \in \mathbf{R}$, the multiplicity $m_{\mathbf{f}, X} \in \mathbb{Z}_{\geq 0}$ of $V(\mathbf{f})$ in any object $X$ of category $\mathcal{O}$ is well-defined, and $[X]:=\sum_{\mathbf{f} \in \mathbf{R}} m_{\mathbf{f}, X}[V(\mathbf{f})] \in K_{0}(\mathcal{O})$. There is no ambiguity for $X=V(\mathbf{f})$ as $m_{\mathbf{n}, V(\mathbf{f})}=\delta_{\mathbf{n}, \mathbf{f}}$ for $\mathbf{n}, \mathbf{f} \in \mathbf{R}$. Make $K_{0}(\mathcal{O})$ into a ring with multiplication induced by $[X][Y]:=[X \otimes Y]$ for $X, Y$ in category $\mathcal{O}$. Since $\chi_{q}$ respects exact sequences and tensor products, the assignment $[X] \mapsto \chi_{q}(X)$, for $X$ in category $\mathcal{O}$, extends uniquely to a ring homomorphism $\chi_{q}: K_{0}(\mathcal{O}) \longrightarrow \mathcal{E}_{\ell}$ called the $q$-character map.

Corollary 6.9. The q-character map $\chi_{q}$ is injective.

Proof. We need to show that $\chi_{q}(V(\mathbf{f}))$ distinguishes f. Indeed, from the proof of Lemma 6.8(1), we deduce that $\chi_{q}(V(\mathbf{f}))$ is $\mathbf{f}$ plus terms of the form $\mathbf{n f}$ where the $\mathbf{n} \in \widehat{\mathfrak{P}}$ satisfy $\varpi(\mathbf{n}) \in q^{\mathbf{Q}_{-}}$. So $\mathbf{f}$ appears in $\chi_{q}(V(\mathbf{f}))$ as a leading term.

Proposition 6.10. Let $\mathbf{f}=\left(\left(f_{i}(z)\right)_{i \in I} ; s\right) \in \mathbf{R}$. Then $V(\mathbf{f})$ is finite-dimensional if and only if for all $i \in I_{0} \backslash\{M\}$ there exist $P_{i}(z) \in 1+z \mathbb{C}[z]$ and $a_{i} \in \mathbb{C}^{\times}$such that $\frac{f_{i}(z)}{f_{i+1}(z)}=a_{i} \frac{P_{i}\left(z q_{i}^{-1}\right)}{P_{i}\left(z q_{i}\right)}$.

Proof. $\left(\right.$ Sketch $\left.^{8}\right)$ Sufficiency: such $\mathbf{f}$ can be written as $h(z) p \mathbf{n}$ where $h(z) \in \mathbb{C}[[z]]^{\times}, p \in \mathfrak{P}$ and $\mathbf{n}$ is a product of the $\mathbf{w}_{a ; q_{r}}^{(r)}, \mathbf{w}_{M, a}^{ \pm}$with $a \in \mathbb{C}^{\times}$and $r \in I_{0}$. So $V(\mathbf{f})$ is a sub-quotient of the tensor product $T$ the $V(h(z) p)$, with the $V\left(\mathbf{w}_{a ; q_{r}}^{(r)}\right)=W_{1, a}^{(r)}$ and $L_{M, a}^{ \pm}$. By Lemma 6.7 and Theorem 3.4, $\operatorname{dim} L_{M, a}^{ \pm}=2^{M N}$. So $T$ and $V(\mathbf{f})$ are finite-dimensional.

\footnotetext{
${ }^{8}$ This result is not needed in the following. We include it here for completeness.
} 
Necessity: let $i \in I_{0}$. One restricts to the subalgebra $Y_{i}$ of $Y_{q}(\mathfrak{g})$ generated by the $s_{j k}^{(n)}, s_{j j}^{-1}$ with $j, k \in\{i, i+1\}$ and $n \geq 0$. It is a quotient algebra of $Y_{q}\left(\mathfrak{g l}_{2}\right)$. The polynomiality can then be proved along the line of [14, Remark 3.11].

The $L_{r, a}^{ \pm}$in [21] are always infinite-dimensional. In our case, by Theorem 3.4 and equation (6.5), $L_{M, a}^{+}$is of dimension $2^{M N}$; see also Example 5.6. We refer to [34, Section 4] for a detailed discussion of finite-dimensional irreducible $Y_{q}(\mathfrak{g l}(1,1))$-modules.

Let $\mathbf{R}_{U}$ be the subset of $\mathbf{R}$ formed of $\mathbf{f}$ satisfying the condition in Lemma $6.8(2)$. The following elements of $\mathbf{R}_{U}$ will be used: let $r \in I_{0}$ and $a \in \mathbb{C}^{\times}$,

$$
A_{r, a}=(\underbrace{1, \ldots, 1}_{r-1}, \frac{q_{r}-z a \theta_{r}^{-1}}{1-z a \theta_{r}^{-1} q_{r}}, \frac{1-z a \theta_{r+1}^{-1} q_{r+1}}{q_{r+1}-z a \theta_{r+1}^{-1}}, \underbrace{1, \ldots, 1}_{M+N-r-1} ;\left|\alpha_{r}\right|) \text {. }
$$

By definition $\varpi\left(A_{r, a}\right)=q^{\alpha_{r}}$. The $A_{r, a}$ generate a free abelian subgroup $\widehat{\mathcal{Q}}$ of $\mathbf{R}_{U}$.

Theorem 6.11. Let $S$ be an irreducible $U_{q}(\widehat{\mathfrak{g}})$-module in category $\mathcal{O}$.

(1) We have $\operatorname{wt}_{\ell}(S) \subseteq \widehat{\mathcal{Q}} \mathbf{f}$ where $\mathbf{f} \in \mathbf{R}_{U}$ is the highest $\ell$-weight of $S$.

(2) If $S$ is finite-dimensional, then in a fraction ring of $K_{0}(\mathcal{O})$,

$$
[S]=\sum_{j=1}^{\operatorname{dim} S}\left[D_{j}\right] \mathbf{m}_{j}
$$

where for each $j, D_{j}$ is a one-dimensional $U_{q}(\widehat{\mathfrak{g}})$-module in category $\mathcal{O}$, and $\mathbf{m}_{j}$ is a product of the $\frac{\left[W_{a ; b}^{(r)}\right]}{\left[W_{a ; c}^{(r)}\right]}$ with $r \in I_{0}$ and $a, b, c \in \mathbb{C}^{\times}$.

(2) can be though of as generalized Baxter's relations in the sense of Frenkel-Hernandez [14, Theorem 4.8]. In equation (6.8), only $U_{q}(\widehat{\mathfrak{g}})$-modules are involved. Proof of (1) and concrete examples are postponed to Section 7 .

Proof of Theorem 6.11(2) assuming (1). Any $\mathbf{n} \in \mathbf{R}_{U}$ can be written as $h(z) p \mathbf{n}^{\prime}$ where $h(z) \in \mathbb{C}[[z]]^{\times}, p=(1, \ldots, 1 ; s) \in \mathfrak{P}$ and $\mathbf{n}^{\prime}$ is a product of the $\frac{\mathbf{w}_{a ; b}^{(r)}}{\mathbf{w}_{a ; c}^{(r)}}=\frac{\chi_{q}\left(W_{a ; b}^{(r)}\right)}{\chi_{q}\left(W_{a ; c}^{(r)}\right)}$; the last identity follows from equation (6.6). By (1), $\mathrm{wt}_{\ell}(S) \subset \mathbf{R}_{U}$. This establishes the $q$-character version of equation (6.8) with isomorphism classes replaced by $q$-characters $\left(D_{j}=V(h(z) p)\right)$. We conclude by the injectivity of the $q$-character map.

Corollary 6.12. The $Y_{q}(\mathfrak{g})$-modules $L_{r, a}^{ \pm}$for $r \in I_{0}$ and $a \in \mathbb{C}^{\times}$are irreducible.

Proof. We show $L_{r, a}^{+}$is irreducible, imitating the proof of [21, Theorem 6.1]. By Definition 5.4 this implies the irreducibility of $L_{r, a}^{-}$. Let $S_{r, a}^{+}$be the sub-module of $L_{r, a}^{+}$generated by its unique highest $\ell$-weight vector. Then $S_{r, a}^{+} \cong V\left(\mathbf{w}_{r, a}^{+}\right)$is irreducible.

We prove that $\widetilde{\chi}_{q}\left(L_{r, a}^{+}\right)=\widetilde{\chi}_{q}\left(S_{r, a}^{+}\right)$. In view of equation (6.6), this means that: if $l>0$ and $\mathbf{w}_{l, a}^{(r)} \mathbf{f} \in \mathrm{wt}_{\ell}\left(W_{l, a}^{(r)}\right)$, then $\operatorname{dim}\left(S_{r, a}^{+}\right)_{\mathbf{w}_{r, a}^{+} \mathbf{f}} \geq \operatorname{dim}\left(W_{l, a}^{(r)}\right)_{\mathbf{w}_{l, a}^{(r)}}$. We shall indicate the dependence of an element $\mathbf{m}=\mathbf{m}(z) \in \widehat{\mathfrak{P}}$ on $z$ whenever necessary. For $\mathbf{f}=1$ this is clear as they are both one-dimensional. Let $\mathbf{f} \neq 1$. By Theorem $6.11(1)$ and equation (6.6), $\mathbf{f} \in \widehat{\mathcal{Q}}$ and $\varpi(\mathbf{f}) \in q^{\mathbf{Q}_{-}}$. The following set $X$ is finite and non-empty:

$$
X:=\left\{\mathbf{n}^{-1} \mathbf{f} \in \widehat{\mathcal{Q}} \mid \mathbf{f} \neq \mathbf{n} \in \widehat{\mathcal{Q}}, \mathbf{w}_{r, a}^{+} \mathbf{n} \in \mathrm{wt}_{\ell}\left(S_{r, a}^{+}\right), \varpi\left(\mathbf{n}^{-1} \mathbf{f}\right) \in q^{\mathbf{Q}_{-}}\right\} \subset \widehat{\mathcal{Q}} \backslash\{1\} .
$$


For $\mathbf{m} \in X$, the set $\left\{\mathbf{m}\left(z q_{r}^{-2 k}\right) \mid k>l\right\}$ is infinite but its intersection with $\mathrm{wt}_{\ell}\left(L_{r, a}^{-}\right)\left(\mathbf{w}_{r, a}^{-}\right)^{-1}$ is finite, because $\varpi\left(\mathbf{m}\left(z q_{r}^{-2 k}\right)\right)=\varpi(\mathbf{m})$ is fixed and weight spaces of $L_{r, a}^{-}$are finite-dimensional. Choose $k>l$ large enough such that $\mathbf{m}\left(z q_{r}^{-2 k}\right) \mathbf{w}_{r, a}^{-} \notin \mathrm{wt}_{\ell}\left(L_{r, a}^{-}\right)$for all $\mathbf{m} \in X$. By Lemma 6.7, $\mathbf{w}_{k, a}^{(r)} \mathbf{f} \in \mathrm{wt}_{\ell}\left(W_{k, a}^{(r)}\right)$. The $Y_{q}(\mathfrak{g})$-module $S_{r, a}^{+} \otimes L_{r, a q_{r}^{2 k}}^{-}$has an irreducible sub-quotient isomorphic to $W_{k, a}^{(r)}$ up to tensor product by one-dimensional $Y_{q}(\mathfrak{g})$-modules. It follows from Lemma 6.4 and Remark 6.5 that $\mathbf{f}=\mathbf{f}_{k}^{+} \mathbf{f}_{k}^{-}$where $\mathbf{w}_{r, a}^{+} \mathbf{f}_{k}^{+} \in \mathrm{wt}_{\ell}\left(S_{r, a}^{+}\right)$and $\mathbf{w}_{r, a q_{r}^{2 k}}^{-} \mathbf{f}_{k}^{-} \in \mathrm{wt}_{\ell}\left(L_{r, a q_{r}^{2 k}}^{-}\right)$, which implies $\mathbf{f}_{k}^{-}\left(z q_{r}^{-2 k}\right) \mathbf{w}_{r, a}^{-} \in \mathrm{wt}_{\ell}\left(L_{r, a}^{-}\right)$based on the pullback of $\Phi_{q_{r}^{2 k}}$ in Proposition 3.2. So $\left(\mathbf{f}_{k}^{+}\right)^{-1} \mathbf{f}=\mathbf{f}_{k}^{-} \notin X$. By Theorem 6.11(1) and equation (6.6), $\mathbf{f}_{k}^{+} \in \widehat{\mathcal{Q}}$ and $\varpi\left(\mathbf{f}_{k}^{-}\right) \in q^{\mathbf{Q}_{-}}$, forcing $\mathbf{f}_{k}^{-}=1$. So any such factorization $\mathbf{f}=\mathbf{f}_{k}^{+} \mathbf{f}_{k}^{-}$is trivial: $\mathbf{f}_{k}^{-}=1$. This proves $\operatorname{dim}\left(W_{l, a}^{(r)}\right)_{\mathbf{w}_{l, a}^{(r)} \mathbf{f}} \leq \operatorname{dim}\left(W_{k, a}^{(r)}\right)_{\mathbf{w}_{k, a}^{(r)} \mathbf{f}} \leq$ $\operatorname{dim}\left(S_{r, a}^{+}\right)_{\mathbf{w}_{r, a}^{+} \mathbf{f}} \cdot$

\section{$7 \quad$ Rationality of $\ell$-weights of finite-dimensional modules}

In this section, we study in more detail the $\ell$-weights of finite-dimensional $U_{q}(\widehat{\mathfrak{g}})$-modules in category $\mathcal{O}$ and prove Theorem $6.11(1)$.

Recall the $U_{q}(\mathfrak{g})$-module $\mathbf{V}$ defined by (3.7). Following [35, Example 2], define the $U_{q}(\mathfrak{g})$ module $\mathbf{W}$ to be the pullback of $\mathbf{V}$ by $\Psi: U_{q}(\mathfrak{g}) \longrightarrow U_{q}(\mathfrak{g})$ in Proposition 3.2. For $i \in I$, set $u_{i}:=\Psi^{*} v_{i} \in \mathbf{W}$; it is of weight $q^{-\epsilon_{i}}$.

For $a \in \mathbb{C}^{\times}$, define the $U_{q}(\widehat{\mathfrak{g}})$-modules $\mathbf{V}(a)$ and $\mathbf{W}(a)$ to be the pullbacks of $\mathbf{V}$ and $\mathbf{W}$ respectively by $\mathrm{ev}_{a}: U_{q}(\widehat{\mathfrak{g}}) \longrightarrow U_{q}(\mathfrak{g})$. Naturally as $Y_{q}(\mathfrak{g})$-modules, $\mathbf{V}(a)$ and $\mathbf{W}(a)$ are in category $\mathcal{O}$, and their weight spaces are always one-dimensional.

Definition 7.1. For $a \in \mathbb{C}^{\times}$and $i \in I$, let $\mathcal{X}_{i, a} \in \mathrm{wt}_{\ell}(\mathbf{V}(a))$ and $\mathcal{Y}_{i, a} \in \mathrm{wt}_{\ell}(\mathbf{W}(a))$ be such that $\varpi\left(\mathcal{X}_{i, a}\right)=q^{\epsilon_{i}}$ and $\varpi\left(\mathcal{Y}_{i, a}\right)=q^{-\epsilon_{i}}$.

Let us compute explicitly the $\mathcal{X}_{i, a}, \mathcal{Y}_{i, a}$, following an idea of [15, Lemma 4.7]. Fix $k, i \in I$. By Definition 7.1, $v_{i}, u_{i}$ are common eigenvectors of $C_{k}(z)$, whose eigenvalues are denoted by $g_{i}^{k}(z), h_{i}^{k}(z) \in \mathbb{C}[[z]]^{\times}$respectively. An important observation from the Gauss decomposition in Section 6 is that: if a vector $x$, either in $\mathbf{V}(a)$ or $\mathbf{W}(a)$ is annihilated by the $s_{j l}^{(n)}, t_{j l}^{(n)}$ for $1 \leq j<l \leq k$, then $K_{j}^{+}(z) x=s_{j j}(z) x$ for $1 \leq j \leq k$. For example, $v_{1}, u_{k}, v_{l}, u_{l}$ for $l>k$ are such vectors according to the weight gradings on $\mathbf{V}, \mathbf{W}$. Therefore for $i>k$,

$$
g_{i}^{k}(z)=\prod_{j=1}^{k}\left(1-z a \theta_{j}\right)^{d_{j}}=h_{i}^{k}(z)
$$

Suppose $i \leq k$. Observe from (3.7) that $v_{i}$ is proportional to $t_{i 1} v_{1}$. Similarly $u_{i}$ is proportional to $t_{k i} u_{k}$; see [35, Example 2]. Since $t_{i 1}, t_{k i}$ commute with $C_{k}(z)$ by Proposition 6.1 , we have $g_{i}^{k}(z)=g_{1}^{k}(z)$ and $h_{i}^{k}(z)=h_{k}^{k}(z)$. We apply the above observation to the vectors $v_{1}, u_{k}$ to compute $g_{1}^{k}(z)$ and $h_{k}^{k}(z)$, and obtain

$$
g_{i}^{k}(z)=\left(q_{1}-z a q_{1}^{-1}\right)^{d_{1}} \prod_{j=2}^{k}\left(1-z a \theta_{j}\right)^{d_{j}}, \quad h_{i}^{k}(z)=\left(q_{k}^{-1}-z a \theta_{k} q_{k}\right)^{d_{k}} \prod_{j=1}^{k-1}\left(1-z a \theta_{j}\right)^{d_{j}} .
$$

It follows that: setting $h(z)=\left(1-z a q^{2}\right)\left(1-z a q^{-2}\right)(1-z a)^{-2}$,

$$
\mathcal{X}_{i, a}=(1-z a) \times(\underbrace{1, \ldots, 1}_{i-1},\left(\frac{q-z a \theta_{i}^{-1} q^{-1}}{1-z a \theta_{i}^{-1}}\right)^{d_{i}}, \underbrace{1, \ldots, 1}_{M+N-i} ;\left|\epsilon_{i}\right|),
$$




$$
\mathcal{Y}_{i, a}=(1-z a) \times(\underbrace{1, \ldots, 1}_{i-1}, \frac{q_{i}^{-1}-z a q_{i}}{1-z a}, \underbrace{h(z), \ldots, h(z)}_{M+N-i} ;\left|\epsilon_{i}\right|) .
$$

We used $\theta_{i+1}=\theta_{i} q_{i} q_{i+1}$ for $i \in I_{0}$. Combining with equation (6.7), we obtain

$$
A_{r, a}=\mathcal{X}_{r, a q} \mathcal{X}_{r+1, a q}^{-1}=\mathcal{Y}_{r, a \theta_{r}^{-1} q_{r}^{-1}}^{-1} \mathcal{Y}_{r+1, a \theta_{r}^{-1} q_{r}^{-1}}
$$

KR modules $W_{1, a}^{(r)}$ can be constructed from $\mathbf{V}, \mathbf{W}$ by a fusion procedure.

Lemma $7.2([35])$. Let $1 \leq s \leq M$ and $1 \leq t<N$. Let $a \in \mathbb{C}^{\times}$. Then as $U_{q}(\widehat{\mathfrak{g}})$-modules,

$$
W_{1, a}^{(s)} \simeq U_{q}(\widehat{\mathfrak{g}}) v_{M+N}^{\otimes s} \subseteq \bigotimes_{1 \leq i \leq s}^{\overleftarrow{\bigotimes}} \mathbf{V}\left(a q^{2 i}\right), \quad W_{1, a}^{(M+N-t)} \simeq U_{q}(\widehat{\mathfrak{g}}) u_{1}^{\otimes t} \subseteq \bigotimes_{1 \leq j \leq t}^{\overleftarrow{ }} \mathbf{W}\left(a q^{2 t-2 j}\right)
$$

Proof. Comparing Definition 3.3 with [35, Lemma 5, Definition 2] we have

$$
W_{1, a}^{(s)}=\mathrm{ev}_{a q^{2}}^{*} L\left(\varpi_{s}\right) \simeq V_{s, a q^{2 s+2}}^{+}:=U_{q}(\widehat{\mathfrak{g}}) v_{M+N}^{\otimes s} \subset \bigotimes_{1 \leq i \leq s}^{\overleftarrow{ }} \mathbf{V}\left(a q^{2 i}\right)
$$

Here we borrow the notation $V_{s, a q^{2 s+2}}^{+}$from loc. cit. The case of $t$ is parallel.

Note that in equation (7.1), $\theta_{r}^{-1} q_{r}^{-1} \in q^{2 \mathbb{Z}+1}$. Let us define $\widehat{\mathcal{Q}}_{a}$ to be the subgroup of $\widehat{\mathcal{Q}}$ generated by the $A_{r, b}$ with $b \in a q^{2 \mathbb{Z}}$ and $r \in I_{0}$.

Corollary 7.3. For $r \in I_{0}, a, c \in \mathbb{C}^{\times}$and $k \in \mathbb{Z}_{>0}$ we have

$$
\left(\mathbf{w}_{k, a}^{(r)}\right)^{-1} \mathrm{wt}_{\ell}\left(W_{k, a}^{(r)}\right) \subseteq\left(\mathbf{w}_{r, a}^{+}\right)^{-1} \mathrm{wt}_{\ell}\left(L_{r, a}^{+}\right)=\left(\mathbf{w}_{a ; c}^{(r)}\right)^{-1} \mathrm{wt}_{\ell}\left(W_{a ; c}^{(r)}\right) \subseteq \widehat{\mathcal{Q}}_{a q} .
$$

Proof. By Lemmas 4.2 and 7.2, there exists a one-dimensional $U_{q}(\widehat{\mathfrak{g}})$-module $D$ such that $D \otimes W_{k, a}^{(r)}$ is a sub-quotient of a tensor product $T$ of the $X(b)$ where $b \in a q^{2 \mathbb{Z}}$ and: if $r \leq M$ then $X=\mathbf{V}$; if $r>M$ then $X=\mathbf{W}$. For any such $X(b)$, let $\mathbf{f}$ be the highest $\ell$-weight of $X(b)$. Then by equation (7.1): $\mathbf{f}^{-1} \mathrm{wt}_{\ell}(X(b)) \subset \widehat{\mathcal{Q}}_{a q}$. Since $\chi_{q}$ respects tensor products, we have $\mathbf{n}^{-1} \mathbf{m} \in \widehat{\mathcal{Q}}_{a q}$ for all $\mathbf{n}, \mathbf{m} \in \mathrm{wt}_{\ell}(T)$. Taking normalized $q$-characters in Remark 6.5 , $\left(\mathbf{w}_{k, a}^{(r)}\right)^{-1} \mathrm{wt}_{\ell}\left(W_{k, a}^{(r)}\right) \subseteq \widehat{\mathcal{Q}}_{a q}$. The rest comes from equation (6.6).

Proof of Theorem 6.11(1). As in the proof of sufficiency of Lemma 6.8(2), the irreducible module $V(\mathbf{f})$ for $\mathbf{f} \in \mathbf{R}_{U}$ can be realized as a sub-quotient of a tensor product $T$ of a onedimensional $U_{q}(\widehat{\mathfrak{g}})$-module $D$ with the $W_{c ; a}^{(r)}$. Comparing normalized $q$-characters, we obtain $(1)$ as a consequence of Corollary 7.3.

Another consequence is the irreducibility of $W_{a ; c}^{(r)}$ for generic $c \in \mathbb{C}^{\times}$.

Corollary 7.4. The $U_{q}(\widehat{\mathfrak{g}})$-module $W_{a ; c}^{(r)}$ is irreducible if $c^{2} \notin q^{2 \mathbb{Z}}$.

Proof. Let $S_{a ; c}^{(r)}$ be the sub-module of $W_{a ; c}^{(r)}$ generated by its highest $\ell$-weight vector; it is irreducible and isomorphic to $V\left(\mathbf{w}_{a ; c}^{(r)}\right)$. Let $k>0$ and $\mathbf{w}_{k, a}^{(r)} \mathbf{f} \in \mathrm{wt}_{\ell}\left(W_{k, a}^{(r)}\right)$. By equation (6.3)

$$
\left(1-z a c^{2}\right) \times \mathbf{w}_{k, a}^{(r)}=\mathbf{w}_{a ; c}^{(r)} \mathbf{w}_{a c^{2} ; c^{-1} q_{r}^{k}}^{(r)} \in \widehat{\mathfrak{P}} .
$$

This implies that $V\left(1-z c^{2}\right) \otimes W_{k, a}^{(r)}$ is an irreducible sub-quotient of $S_{a ; c}^{(r)} \otimes W_{a c^{2} ; c^{-1} q_{r}^{k}}^{(r)}$. Taking normalized $q$-characters and noting that $V\left(1-z a c^{2}\right)$ is one-dimensional, we have $\mathbf{f}=\mathbf{f}_{+} \mathbf{f}_{-}$where 
$\mathbf{w}_{a ; c}^{(r)} \mathbf{f}_{+} \in \mathrm{wt}_{\ell}\left(W_{a ; c}^{(r)}\right)$ and $\mathbf{w}_{a c^{2} ; c^{-1} q_{r}^{k}}^{(r)} \mathbf{f}_{-} \in \mathrm{wt}_{\ell}\left(W_{a c^{2} ; c^{-1} q_{r}^{k}}^{(r)}\right)$. By Corollary 7.3, we have $\mathbf{f}, \mathbf{f}_{+} \in \widehat{\mathcal{Q}}_{a q}$ and $\mathbf{f}_{-} \in \widehat{\mathcal{Q}}_{a c^{2} q}$. The assumption on $c$ implies that $\widehat{\mathcal{Q}}_{a q} \cap \widehat{\mathcal{Q}}_{a c^{2} q}=\{1\}$, which forces $\mathbf{f}_{-}=1$. So any such factorization $\mathbf{f}=\mathbf{f}_{+} \mathbf{f}_{-}$is trivial: $\mathbf{f}_{-}=1$. This shows that $\operatorname{dim}\left(W_{k, a}^{(r)}\right)_{\mathbf{w}_{k, a}^{(r)} \mathbf{f}} \leq \operatorname{dim}\left(S_{a ; c}^{(r)}\right)_{\mathbf{w}_{a ; c}^{(r)} \mathbf{f}}$ for all $k$, and therefore $S_{a ; c}^{(r)}=W_{a ; c}^{(r)}$.

Example 7.5 (Baxter's relations for $\mathfrak{g l}(2,1))$. We derive equation $(6.8)$ for the $U_{q}(\widehat{\mathfrak{g}})$-modules $\mathbf{V}(a)$ and $\mathbf{W}(a)$, along the line of the proof of Theorem $6.11(2)$ :

$$
\begin{aligned}
& \frac{\mathbf{w}_{a ; c}^{(1)}}{\mathbf{w}_{a ; 1}^{(1)}}=\left(c, \frac{1-z a c^{2}}{1-z a}, \frac{1-z a c^{2}}{1-z a} ; \overline{0}\right), \quad \frac{\mathbf{w}_{a ; c}^{(2)}}{\mathbf{w}_{a ; 1}^{(2)}}=\left(c, c, \frac{1-z a c^{2}}{1-z a} ; \overline{0}\right), \\
& \mathcal{X}_{1, a}=(1-z a) \times\left(\frac{q-z a q^{-1}}{1-z a}, 1,1 ; \overline{0}\right)=\left(1-z a q^{-2}\right) \frac{\mathbf{w}_{a q^{-2} ; q}^{(1)}}{\mathbf{w}_{a q^{-2} ; 1}^{(1)}}, \\
& \mathcal{X}_{2, a}=(1-z a) \times\left(1, \frac{q-z a q^{-3}}{1-z a q^{-2}}, 1 ; \overline{0}\right)=(1-z a) \frac{\mathbf{w}_{a q^{-2} ; q^{-1}}^{(1)}}{\mathbf{w}_{a q^{-2} ; 1}^{(1)}} \frac{\mathbf{w}_{a q^{-4} ; q}^{(2)}}{\mathbf{w}_{a q^{-4} ; 1}^{(2)}}, \\
& \mathcal{X}_{3, a}=(1-z a)\left(1,1, \frac{1-z a q^{-2}}{q-z a q^{-3}} ; \overline{1}\right)=(1-z a) \frac{\mathbf{w}_{a q^{-4} ; q}^{(2)}}{\mathbf{w}_{a q^{-4} ; 1}^{(2)}}\left(q^{-1}, q^{-1}, q^{-1} ; \overline{1}\right) \text {, } \\
& \mathcal{Y}_{1, a}=\left(q^{-1}-z a q, \frac{\left(1-z a q^{2}\right)\left(1-z a q^{-2}\right)}{1-z a}, \frac{\left(1-z a q^{2}\right)\left(1-z a q^{-2}\right)}{1-z a} ; \overline{0}\right) \\
& =\left(1-z a q^{2}\right) \frac{\mathbf{w}_{a ; q^{-1}}^{(1)}}{\mathbf{w}_{a ; 1}^{(1)}}, \\
& \mathcal{Y}_{2, a}=\left(1-z a, q^{-1}-z a q, \frac{\left(1-z a q^{2}\right)\left(1-z a q^{-2}\right)}{1-z a} ; \overline{0}\right)=(1-z a) \frac{\mathbf{w}_{a ; q}^{(1)}}{\mathbf{w}_{a ; 1}^{(1)}} \frac{\mathbf{w}_{a ; q^{-1}}^{(2)}}{\mathbf{w}_{a ; 1}^{(2)}} \\
& \mathcal{Y}_{3, a}=(1-z a) \times\left(1,1, \frac{q-z a q^{-1}}{1-z a} ; \overline{1}\right)=(1-z a) \frac{\mathbf{w}_{a ; q^{-1}}^{(2)}}{\mathbf{w}_{a ; 1}^{(2)}}(q, q, q ; \overline{1}) \text {. }
\end{aligned}
$$

The irreducible $U_{q}(\widehat{\mathfrak{g}})$-module of highest $\ell$-weight $(g(z), g(z), g(z) ; s)$ being one-dimensional for $g(z) \in \mathbb{C}[[z]]^{\times}$and $s \in \mathbb{Z}_{2}$, its isomorphism class is denoted by $[g(z) s]$. Then

$$
\begin{aligned}
& {\left[\mathbf{V}\left(a q^{2}\right)\right]=[1-z a] \frac{\left[W_{a ; q}^{(1)}\right]}{\left[W_{a ; 1}^{(1)}\right]}+\left[1-z a q^{2}\right] \frac{\left[W_{a ; q^{-1}}^{(1)}\right]}{\left[W_{a ; 1}^{(1)}\right]} \frac{\left[W_{a q^{-2} ; q}^{(2)}\right]}{\left[W_{a q^{-2} ; 1}^{(2)}\right]}+\left[\left(q^{-1}-z a q\right) \overline{1}\right] \frac{\left[W_{a q^{-2} ; q}^{(2)}\right]}{\left[W_{a q^{-2} ; 1}^{(2)}\right]},} \\
& {[\mathbf{W}(a)]=\left[1-z a q^{2}\right] \frac{\left[W_{a ; q^{-1}}^{(1)}\right]}{\left[W_{a ; 1}^{(1)}\right]}+[1-z a] \frac{\left[W_{a ; q}^{(1)}\right]}{\left[W_{a ; 1}^{(1)}\right]} \frac{\left[W_{a ; q^{-1}}^{(2)}\right]}{\left[W_{a ; 1}^{(2)}\right]}+[(q-z a q) \overline{1}] \frac{\left[W_{a ; q^{-1}}^{(2)}\right]}{\left[W_{a ; 1}^{(2)}\right]} .}
\end{aligned}
$$

To derive Baxter's relations, one needs to compute its $q$-character of finite-dimensional $U_{q}(\widehat{\mathfrak{g}})$ modules. In a previous version of this paper (arXiv:1410.0837v2), the author was able to prove a tableau-sum formula for $\chi_{q}\left(\mathrm{ev}_{a}^{*} L(\lambda)\right)$, with $L(\lambda)$ being an irreducible submodule of a tensor power of $\mathbf{V}$ (polynomial modules), based on an idea of [15] relating $\ell$-weights to GelfandTsetlin basis [26]. Such formula appeared earlier in the context of transfer matrices of quantum integrable systems attached to $U_{q}(\widehat{\mathfrak{g}})$ [30]. In a recent preprint [36], the tableau-sum $q$-character formula has been extended to

$$
\mathrm{ev}_{a}^{*}\left(L^{*}(\lambda)\right), \quad\left(\mathrm{ev}_{a}^{\prime}\right)^{*} L(\lambda), \quad\left(\mathrm{ev}_{a}^{\prime}\right)^{*}\left(L^{*}(\lambda)\right)
$$


where $\mathrm{ev}_{a}^{\prime}$ is a second evaluation map arising from involution and $L^{*}(\lambda)$ is the dual module. To avoid redundancy, in the present paper we do not present the $q$-character formula.

\section{A Non-twisted quantum loop algebras}

We apply the asymptotic construction $\left(c \in \mathbb{C}^{\times}\right)$of Section 2 to the inductive system in [21] of KR modules over an arbitrary non-twisted quantum loop algebra.

The main step is to establish the asymptotic property as Lemma 5.1. In Section 5, we used the evaluation maps to reduce to the finite-type quantum supergroup (Proposition 4.3). What is essential is the representation theory of $U_{q}\left(\mathfrak{s l}_{2}\right)$ and $U_{q}(\mathfrak{s l}(1,1))$. The evaluation maps, which do not exist for quantum loop algebras out of type A, do not play big rôle.

We use freely the notations of [21], and ignore those from Sections 3-7. The quantum loop algebra $U_{q}(\mathfrak{g})$ admits Drinfeld generators $x_{i, r}^{ \pm}, \phi_{i, \pm m}^{ \pm}$for $i \in I, r \in \mathbb{Z}$ and $m \in \mathbb{Z}_{\geq 0}$; see [21, equation (2.2)]. ( $\mathfrak{g}$ here is $\widehat{\mathfrak{a}}$ in the introduction.) We shall need the following basic facts.

(A) Algebra $U_{q}(\mathfrak{g})$ is generated by $S:=\left\{\phi_{i, \pm m}^{ \pm}, x_{i, r}^{+}, x_{i, 0}^{-} \mid i \in I, m \in \mathbb{Z}_{\geq 0}, r \in \mathbb{Z}\right\}$.

(B) For $i \in I,\left(x_{i, 0}^{+}, x_{i, 0}^{-}, \phi_{0}^{+}\right)$generates $U_{q_{i}}\left(\mathfrak{s l}_{2}\right)$ with coproduct:

$$
\begin{aligned}
& \Delta\left(x_{i, 0}^{+}\right)=\phi_{i, 0}^{+} \otimes x_{i, 0}^{+}+x_{i, 0}^{+} \otimes 1, \quad \Delta\left(x_{i, 0}^{-}\right)=1 \otimes x_{i, 0}^{-}+x_{i, 0}^{-} \otimes \phi_{i, 0}^{-}, \\
& \Delta\left(\phi_{i, 0}^{+}\right)=\phi_{i, 0}^{+} \otimes \phi_{i, 0}^{+} .
\end{aligned}
$$

Let us be in the situation of [21, Section 4.2] where an inductive system of $\mathrm{KR}$ modules $\left(F_{k, l}: V_{l} \longrightarrow V_{k}\right)_{l<k}$ for a fixed $i \in I$ was constructed. For $j \in I, r \in \mathbb{Z}$ and $m \in \mathbb{Z}_{\geq 0}$, the $F_{k, l}$ commute with the $x_{j, r}^{+}$, and the $\phi_{j, \pm m}^{ \pm} F_{k, l}$ for $k>l$, as Laurent polynomials in $q_{i}^{k}$ is described in [21, Proposition 4.2]. It is therefore enough to study the $x_{j, 0}^{-} F_{k, l}$ for $k>l$.

If $j \neq i$, then $x_{j, 0}^{-}$annihilates the highest $\ell$-weight vector of $V_{k}$. This gives $x_{j, 0}^{-} F_{k, l}=F_{k, l} x_{j, 0}^{-}$. Assume $j=i$. The structural maps $F_{k, l}$ come from $U_{q}(\mathfrak{g})$-linear maps in [21, Theorem 3.15] which we write as $\mathscr{F}_{k, l}: V_{l} \otimes Z_{l k} \longrightarrow V_{k}$, where $Z_{l k}:=L\left(M_{k}^{\leq(-2 l+1) d_{i}}\right)$ before [21, equation (4.26)] with highest $\ell$-weight vector $v_{l k}$ fixed so that $v_{l} \otimes v_{l k} \mapsto v_{k}$.

Claim A.1. Let $v \in V_{l}$. Then for $k>l+1$, we have $x_{i, 0}^{-} F_{k, l}(v) \in F_{k, l+1}\left(V_{l+1}\right)$ with

$$
x_{i, 0}^{-} F_{k, l}(v)=F_{k, l+1}\left(q_{i}^{l-k} F_{l+1, l}\left(x_{i, 0}^{-} v\right)+\frac{q_{i}^{k-l}-q_{i}^{l-k}}{q_{i}-q_{i}^{-1}} \mathscr{F}_{l+1, l}\left(v \otimes x_{i, 0}^{-} v_{l, l+1}\right)\right) .
$$

The proof is the same as that of Proposition 4.3(3), based on (B), and the fact that for $j \in I$ the weight space of $V_{k}$ of weight $\bar{\omega}_{i}^{k} \bar{\alpha}_{j}^{-1}$ is of dimension $\delta_{j i}$.

The asymptotic property of Section 2 is established for the generating set $S$ of $U_{q}(\mathfrak{g})$. One can then form a representation $\rho_{c}$ of $U_{q}(\mathfrak{g})$, with $c \in \mathbb{C}^{\times}$on the limit $V_{\infty}$ by specializing Laurent polynomials in $q_{i}^{k}$ to $c$. The representation $\rho_{c}$ contains a unique (up to multiple) vector annihilated by the $x_{j, r}^{+}$; it is $v_{\infty}$, the inductive limit of highest $\ell$-weight vectors $v_{k} \in V_{k}$. Moreover, we obtain from the proof of [21, Proposition 4.2] that

$$
\phi_{j}^{ \pm}(z) v_{\infty}=v_{\infty} \quad \text { for } \quad j \neq i, \quad \phi_{i}^{ \pm}(z) v_{\infty}=\frac{c-z a c^{-1}}{1-z a} v_{\infty} .
$$

The normalized $q$-character of $\rho_{c}$ is identical to that of $L_{i, a}^{-}$in [21, Theorem 6.1]. The representation $\rho_{c}$ belongs to category $\mathcal{O}$ of $U_{q}(\mathfrak{g})$-modules $[19,25]$.

In [21, Lemma 4.4, Proposition 4.5], asymptotic property for the $x_{j, r}^{-} F_{k, l}$ was deduced from delicate results on $q$-characters of representations [20]. 
The arguments should work for Yangians: inductive systems of KR modules come from cyclicity result of particular tensor products of fundamental modules [29]; their asymptotic property is reduced eventually to representation theory of $\mathfrak{s l}_{2}$. See [13, Appendix A] for the case of Yangian of $\mathfrak{s l}_{2}$. Alternatively one may start from asymptotic modules $\left(\rho_{c}, V_{\infty}\right)$ of a quantum loop algebra and transform them into modules over the Yangian via the functor of GautamToledano Laredo [17, Section 6].

\section{Acknowledgements}

The author thanks Vyjayanthi Chari, Giovanni Felder, David Hernandez, Masaki Kashiwara, Eugene Mukhin, Zengo Tsuboi, and Weiqiang Wang for interesting discussions. He is supported by the National Center of Competence in Research SwissMAP - The Mathematics of Physics of the Swiss National Science Foundation.

\section{References}

[1] Baxter R.J., Partition function of the eight-vertex lattice model, Ann. Physics 70 (1972), 193-228.

[2] Bazhanov V.V., Hibberd A.N., Khoroshkin S.M., Integrable structure of $\mathcal{W}_{3}$ conformal field theory, quantum Boussinesq theory and boundary affine Toda theory, Nuclear Phys. B 622 (2002), 475-547, hep-th/0105177.

[3] Bazhanov V.V., Lukyanov S.L., Integrable structure of quantum field theory: classical flat connections versus quantum stationary states, J. High Energy Phys. 2014 (2014), no. 9, 147, 68 pages, arXiv:1310.4390.

[4] Bazhanov V.V., Lukyanov S.L., Zamolodchikov A.B., Integrable structure of conformal field theory, quantum KdV theory and thermodynamic Bethe ansatz, Comm. Math. Phys. 177 (1996), 381-398, hep-th/9412229.

[5] Bazhanov V.V., Lukyanov S.L., Zamolodchikov A.B., Integrable structure of conformal field theory. II. Qoperator and DDV equation, Comm. Math. Phys. 190 (1997), 247-278, hep-th/9604044.

[6] Bazhanov V.V., Lukyanov S.L., Zamolodchikov A.B., Integrable structure of conformal field theory. III. The Yang-Baxter relation, Comm. Math. Phys. 200 (1999), 297-324, hep-th/9805008.

[7] Bazhanov V.V., Tsuboi Z., Baxter's Q-operators for supersymmetric spin chains, Nuclear Phys. B 805 (2008), 451-516, arXiv:0805.4274.

[8] Beisert N., Galleas W., Matsumoto T., A quantum affine algebra for the deformed Hubbard chain, J. Phys. A: Math. Theor. 45 (2012), 365206, 20 pages, arXiv:1102.5700.

[9] Benkart G., Kang S.-J., Kashiwara M., Crystal bases for the quantum superalgebra $U_{q}(\mathfrak{g l}(m, n))$, J. Amer. Math. Soc. 13 (2000), 295-331, math.QA/9810092.

[10] Damiani I., La $R$-matrice pour les algèbres quantiques de type affine non tordu, Ann. Sci. École Norm. Sup. (4) 31 (1998), 493-523.

[11] Faddeev L.D., Reshetikhin N.Yu., Takhtajan L.A., Quantization of Lie groups and Lie algebras, in YangBaxter equation in Integrable Systems, Advanced Series in Mathematical Physics, Vol. 10, Editor M. Jimbo, World Scientific Publishing Co., Inc., Teaneck, NJ, 1989, 299-309.

[12] Felder G., Elliptic quantum groups, in XIth International Congress of Mathematical Physics (Paris, 1994), Int. Press, Cambridge, MA, 1995, 211-218, hep-th/9412207.

[13] Felder G., Zhang H., Baxter operators and asymptotic representations, Selecta Math. (N.S.), to appear, arXiv:1611.00628.

[14] Frenkel E., Hernandez D., Baxter's relations and spectra of quantum integrable models, Duke Math. J. 164 (2015), 2407-2460, arXiv:1308.3444.

[15] Frenkel E., Mukhin E., The Hopf algebra Rep $U_{q} \widehat{\mathfrak{g l}}_{\infty}$, Selecta Math. (N.S.) 8 (2002), 537-635, math.QA/0103126.

[16] Frenkel E., Reshetikhin N., The $q$-characters of representations of quantum affine algebras and deformations of $\mathcal{W}$-algebras, in Recent Developments in Quantum Affine Algebras and Related Topics (Raleigh, NC, 1998), Contemp. Math., Vol. 248, Amer. Math. Soc., Providence, RI, 1999, 163-205, math.QA/9810055.

[17] Gautam S., Toledano Laredo V., Yangians, quantum loop algebras, and abelian difference equations, J. Amer. Math. Soc. 29 (2016), 775-824, arXiv:1310.7318. 
[18] Heckenberger I., Spill F., Torrielli A., Yamane H., Drinfeld second realization of the quantum affine superalgebras of $D^{(1)}(2,1 ; x)$ via the Weyl groupoid, in Combinatorial Representation Theory and Related Topics, RIMS Kôkyûroku Bessatsu, Vol. B8, Res. Inst. Math. Sci. (RIMS), Kyoto, 2008, 171-216, arXiv:0705.1071.

[19] Hernandez D., Representations of quantum affinizations and fusion product, Transform. Groups 10 (2005), 163-200, math.QA/0312336.

[20] Hernandez D., Simple tensor products, Invent. Math. 181 (2010), 649-675, arXiv:0907.3002.

[21] Hernandez D., Jimbo M., Asymptotic representations and Drinfeld rational fractions, Compos. Math. 148 (2012), 1593-1623, arXiv:1104.1891.

[22] Ip I.C.-H., Zeitlin A.M., Q-operator and fusion relations for $U_{q}\left(C^{(2)}(2)\right)$, Lett. Math. Phys. 104 (2014), 1019-1043, arXiv:1312.4063.

[23] Kac V.G., Infinite-dimensional Lie algebras, 3rd ed., Cambridge University Press, Cambridge, 1990.

[24] Kulish P.P., Zeitlin A.M., Superconformal field theory and SUSY $N=1 \mathrm{KdV}$ hierarchy. II. The $Q$-operator, Nuclear Phys. B $\mathbf{7 0 9}$ (2005), 578-591, hep-th/0501019.

[25] Mukhin E., Young C.A.S., Affinization of category $\mathcal{O}$ for quantum groups, Trans. Amer. Math. Soc. 366 (2014), 4815-4847, arXiv:1204.2769.

[26] Palev T.D., Stoilova N.I., Van der Jeugt J., Finite-dimensional representations of the quantum superalgebra $U_{q}[\mathrm{gl}(n / m)]$ and related $q$-identities, Comm. Math. Phys. 166 (1994), 367-378, hep-th/9306149.

[27] Perk J.H.H., Schultz C.L., New families of commuting transfer matrices in $q$-state vertex models, Phys. Lett. A 84 (1981), 407-410.

[28] Reshetikhin N.Yu., Takhtadzhyan L.A., Faddeev L.D., Quantization of Lie groups and Lie algebras, Leningrad Math. J. 1 (1990), 193-225.

[29] Tan Y., Guay N., Local Weyl modules and cyclicity of tensor products for Yangians, J. Algebra 432 (2015), 228-251, arXiv:1503.06510.

[30] Tsuboi Z., Analytic Bethe ansatz and functional equations for Lie superalgebra $\operatorname{sl}(r+1 \mid s+1)$, J. Phys. A: Math. Gen. 30 (1997), 7975-7991, arXiv:0911.5386.

[31] Tsuboi Z., Asymptotic representations and $q$-oscillator solutions of the graded Yang-Baxter equation related to Baxter Q-operators, Nuclear Phys. B 886 (2014), 1-30, arXiv:1205.1471.

[32] Yamane H., Quantized enveloping algebras associated with simple Lie superalgebras and their universal R-matrices, Publ. Res. Inst. Math. Sci. 30 (1994), 15-87.

[33] Zhang H., Representations of quantum affine superalgebras, Math. Z. 278 (2014), 663-703, arXiv:1309.5250.

[34] Zhang H., RTT realization of quantum affine superalgebras and tensor products, Int. Math. Res. Not. 2016 (2016), 1126-1157, arXiv:1407.7001.

[35] Zhang H., Fundamental representations of quantum affine superalgebras and $R$-matrices, Transform. Groups 22 (2017), 559-590, arXiv:1506.06093.

[36] Zhang H., Length two representations of quantum affine superalgebras and Baxter operators, arXiv:1612.09476.

[37] Zhang H., Elliptic quantum groups and Baxter relations, arXiv:1706.07574. 